Revue européenne des sciences sociales

European Journal of Social Sciences

XL-124 | 2002

Histoire, philosophie et sociologie des sciences

\title{
Objectivité et subjectivité en science. Quelques aperçus
}

Jacqueline Feldman

\section{(2) OpenEdition}

\section{Journals}

Édition électronique

URL : http://journals.openedition.org/ress/577

DOI : $10.4000 /$ ress. 577

ISSN : 1663-4446

Éditeur

Librairie Droz

Édition imprimée

Date de publication : 1 août 2002

Pagination : 85-130

ISBN : 2-600-00806-3

ISSN : 0048-8046

\section{Référence électronique}

Jacqueline Feldman, "Objectivité et subjectivité en science. Quelques aperçus », Revue européenne des sciences sociales [En ligne], XL-124 | 2002, mis en ligne le 01 décembre 2009, consulté le 10 décembre 2020. URL : http://journals.openedition.org/ress/577 ; DOI : https://doi.org/10.4000/ress. 577

(c) Librairie Droz 


\section{OBJECTIVITÉ ET SUBJECTIVITÉ EN SCIENCE. QUELQUES APERÇUS}

\section{INTRODUCTION}

La science a obtenu la place privilégiée qu'elle occupe dans notre société parce qu'elle était en mesure de fournir des connaissances objectives. Ceci la distinguait de la philosophie, dont elle est issue pourtant - «philosophie naturelle» est un terme encore utilisé au $19^{\text {ème }}$ siècle.

Remarquons que, si on substitue au terme «objectivité » le terme moins fort de «consensus», on se trouve en présence de la question première de toute société démocratique : comment arriver à établir des accords forts entre individus tous différents a priori. La science classique a obtenu une solution partielle, qui porte sur la connaissance, en donnant les moyens d'une recherche collective, qui a remporté de tels succès qu'ils ont bouleversé - et continuent de bouleverser, à travers la technique, nos modes de vie. Mais cette solution n'est pas de nature vraiment démocratique, puisqu'elle a été obtenue par une séparation de la cité scientifique et de ses experts d'avec le monde extérieur, profane.

En raison de ses succès, une assimilation a été faite entre science et progrès les progrès de la science étant censés certifier ceux de la société tout entière. Comme on le sait, on a assisté, ces dernières décennies, à des remises en cause de ce qui avait longtemps paru à la plupart une évidence. Dans une réaction habituelle, des tendances sceptiques ont succédé au dogmatisme du scientisme.

Dans la suspicion exercée désormais par beaucoup envers la science, on peut déceler deux causes, a priori opposées, ce qui est paradoxal: d'une part, les succès mêmes de la science et de la technique qui l'accompagne étroitement ont conduit nos sociétés à des dangers, d'ordre chimique, nucléaire, écologique, biologique... La puissance de la science a été obtenue par une focalisation de l'esprit éthique à l'intérieur de sa propre démarche, en abandonnant les problèmes de ses retombées dans la société et dans la nature.

D'autre part, toujours en raison de ses succès, on a voulu étendre la démarche scientifique à tous les domaines, y compris ceux pour lesquels elle peine à établir des résultats probants, des expertises reconnues, soit en raison de la complexité des situations - le réchauffement du climat, par exemple -, soit, comme c'est le cas pour les sciences humaines et sociales, qu'elle participe à l'évolution même de ses «objets d'étude». La confiance que l'on peut faire à ces types d'expertise relève à la fois de problèmes épistémologiques, éthiques et politiques.

La mise en question de la science a conduit certains sociologues à insister sur ses assises historiques et sociales. Des penseurs post-modernes et/ou féministes, surtout américains, ont relevé qu'elle est le fait de catégories spécifiques - 
essentiellement des hommes des sociétés occidentales. Contre l'imagerie d'une méthode scientifique bien établie, l' «anarchiste» Paul Feyerabend lance, dans les années soixante-dix, son «everything goes». Le livre de Kuhn, La structure des révolutions scientifiques, avec son thème du paradigme, fait ressortir certains de ses caractères sociaux, et connaît un large succès auprès des sociologues enclins au relativisme, succès non dénué de malentendus que Kuhn dénoncera plus tard. Notons que la radicalité de certaines positions a eu pour résultat positif de susciter des réflexions plus approfondies.

Dans cet article, je me propose d'examiner certains aspects subjectifs de la science, en me concentrant sur le fait qu'elle est fabriquée par des personnes. La subjectivité annoncée porte sur la mise en valeur de certains traits qui relèvent explicitement du caractère humain de ceux qui la font. Ce qui, il va sans dire, ne remet pas en cause l'objectivité dont elle peut se prévaloir dans certains domaines, essentiellement les sciences qui ont été, pour cela, dites «exactes», et que je différentierai des sciences sociales, où la part de subjectivité doit être approchée de façon différente, sans oublier pourtant qu'il s'agit, dans les deux cas, de projets de connaissance.

C'est en 1958 que le physicien devenu philosophe Michael Polanyi publie un livre important, non traduit à ma connaissance, intitulé Personal Knowledge $e^{1}$. Dans ce travail qui porte sur la «nature et la justification du savoir scientifique », l'auteur commence par défendre l'idée que tout savoir est personnel, y compris donc, celui de la science: savoir, personnel, ces deux mots apparaissaient à l'époque comme contradictoires, comme le note Polanyi, qui, au début de la préface, pose son refus du scientisme dominant:

Je commence par rejeter l'idéal du détachement scientifique. Dans les sciences exactes, cet idéal ne fait peut-être pas de mal, car, de fait, les scientifiques n'en tiennent pas compte. Mais nous verrons qu'il exerce une influence destructive en biologie, psychologie et sociologie, et pervertit notre entière perspective bien au-delà du domaine de la science.

Mais notre auteur ne donne pas pour autant dans le relativisme: un savoir personnel n'est pas nécessairement un savoir subjectif.

Telle est la participation personnelle de celui qui connaît dans tous les actes de compréhension. Mais ceci ne rend pas pour autant notre entendement subjectif. La compréhension n'est ni un acte arbitraire ni une expérience passive, mais un acte responsable qui prétend à une validité universelle. Ce type de connaissance est donc objective dans le sens où elle établit un contact avec une réalité cachée.

Enfin, l'auteur note ce qu'il y a d'engagement personnel dans toute démarche de connaissance réfléchie, ce qu'on nommerait aujourd'hui, implication:

Le savoir personnel est un engagement intellectuel... Dans tout acte de connaissance, il entre une contribution passionnée de la personne qui connaît ce qui est en train d'être connu.

1 M. Polanyi, Personal Knowledge, Towards a Post-Critical Philosophy, London, Routledge \& Kegan Paul, 1958. 
Dans cet examen de certains traits subjectifs du scientifique, je me trouve rejoindre une autre tendance importante, et sur le long terme, de notre société: l'intérêt toujours plus fort et précis qu'elle porte à la personne. Je citerai, en guise d'illustration, les principes des droits de l'homme, le développement de la psychanalyse et des diverses techniques psychothérapeutiques, le remplacement de la morale par l'éthique, vue comme un choix plus personnel...

Je tiens cependant à souligner que je crois en une certaine autonomie de la pensée, suivant en cela, par exemple, la classification que fait Popper en trois mondes des divers «objets» existant, dont le troisième contient «le monde de l'esprit humain».

Ce travail ne prétend à aucune exhaustivité ni réflexion générale, il n'a d'autre ambition que faire ressortir quelques éléments que j'ai rencontrés et qui me paraissent relever des spécificités des deux types de science que je considère.

\title{
I. LA PRÉSENCE DU SUJET DANS LES SCIENCE EXACTES
}

\begin{abstract}
Il me semble exigible de toute réflexion sur la science qu'elle soit, entre autre chose, une célébration de la pensée scientifique comme telle... l'école pourvoyeuse de méthode, de vérité et de conceptualisation inouïes qu'est la science.
\end{abstract}

Jean-Michel Salanskis ${ }^{2}$

Ces sciences nous apportent des résultats qui sont universels parce qu'ils sont objectifs, parfaitement détachés de ceux qui les ont obtenus, reproductibles, pouvant ainsi faire l'objet d'exposés neutres. On en oublierait qu'ils ont été «découverts », ou «construits », par des personnes concrètes, et dans des contextes également particuliers ${ }^{3}$.

Et pourtant, on sait bien que l'entreprise de production de la science est pleine d'aventures, puisqu'il va s'agir de découvertes, pleine de passions, passions contrôlées par les contraintes de la démarche, faite d'invention, de rigueur, de curiosité, de logique. Aussi le sujet qui fait la science y est étonnamment présent. L'objectivité se construit difficilement, avec lenteur, dans les «essais et erreurs».

C'est en revenant au tout début de la science moderne qu'on peut le mieux saisir ce qui fait, dans l'attitude intellectuelle des premiers savants, la spécificité de cette démarche scientifique qui va s'imposer.

2 Un auteur plutôt «anti-sokalien» J.-M. Salanskis, Pour une épistémologie de la lecture, in B. Jurdant, Impostures scientifiques; Les malentendus de l'affaire Sokal, Paris/Nice, La découverte /Alliage, 1998, p. 163.

3 La science, phénomène social, ne peut se développer que dans une société prête à l'accepter et à l'encourager, en distribuant crédits, salaires, bourses et récompenses. 


\title{
Les débuts : une «révolution spirituelle»
}

\begin{abstract}
Maintes et maintes fois, en étudiant l'histoire de la pensée philosophique et scientifique du $\mathrm{XVI}^{\mathrm{e}}$ et du XVII ${ }^{\mathrm{e}}$ siècle - elles sont en effet si étroitement entremêlées et liées ensemble que, séparées, elles deviennent incompréhensibles - j'ai été forcé de constater, comme beaucoup d'autres l'ont fait avant moi, que, pendant cette période, l'esprit humain ou, tout au moins, l'esprit européen, a subi - ou accompli - une révolution spirituelle très profonde, révolution qui modifia les fondements et les cadres mêmes de notre pensée, et dont la science moderne est à la fois la racine et le fruit.
\end{abstract}

C'est ainsi qu'Alexandre Koyré commence son livre intitulé Du monde clos à l' univers infini ${ }^{4}$, où il nous fait suivre le développement de cette révolution. Ainsi, pour faire ressortir l'importance du changement qui s'opère, Koyré fait appel, non pas au terme «intellectuel», comme je viens de le faire moi-même plus haut, mais au terme beaucoup plus fort de spiritualité.

Voilà qui peut sembler paradoxal: de nos jours de grande laïcité - un des résultats même de la révolution scientifique - ce terme est plus volontiers lié à l'idée de religion qu'à l'idée de science, celle-ci s'opposant souvent à celle-là et ayant réussi à la marginaliser, au moins dans les démarches de connaissance. Mais si l'on se replace aux débuts de la science, ressort clairement la nouveauté d'un type d'esprit qui concerne les modes de pensée et de connaissance, mais aussi qui est basé sur des positions ontologiques plus ou moins bien perçues par les acteurs eux-mêmes. Ces attitudes concernent une grande partie de leur personnalité: le choix de la rationalité, de l'observation attentive, de l'esprit de critique engage profondément ceux qui la font, ce dont on s'aperçoit d'autant plus qu'ils sont encore minoritaires.

L'institution de la science n'existe pas encore, qui permet aujourd'hui la formation des jeunes esprits. C'est dire qu'aucun de ces aventuriers des nouvelles façons de penser ne trouve de voie toute tracée, et chacun va peu à peu dégager la sienne. Chacun à sa manière propre, mais qui devait contribuer à la construction commune, s'efforce de définir la «vraie manière de philosopher», celle qui se distingue des «bavardages » habituels des philosophes, et qui peut conduire à établir des connaissances solides, en utilisant l'observation systématique, le raisonnement mathématique, mais aussi l'invention de techniques multipliant de façon inouïe les possibilités d'observation et d'action sur le réel.

Galilée est un de ceux qui est le plus conscient de ces nouvelles manières de philosopher. S'il représente pour beaucoup le premier scientifique moderne, c'est qu'en plus de l'innovation technique qu'il apporte, des observations qu'il fait, des résultats qu'il obtient, de la quantification qu'il défend, il est le premier savant épistémologue, s'efforçant constamment de présenter les nouvelles manières de penser et de les justifier. Il veut ouvrir un autre monde de connaissance, qui se fonde sur «des expériences sensibles et des raisonnements nécessaires». Après tout, plaide-t-il, si Dieu nous a donné la capacité de raisonner, c'est pour nous en servir et pas seulement se soumettre aux autorités. Grâce à la lunette astronomique qu'il fabrique, il est en mesure d'observer les astres dans le ciel de façon beaucoup plus précise. Voilà qui permet d'en finir avec «les disputes qui ont tourmenté les

\footnotetext{
4 A. Koyré, Du monde clos à l' univers infini, Paris, PUF, 1962.
} 
philosophes pendant tant de siècles ». «La certitude qu'apportent les yeux » permet de se «libérer de verbeuses discussions ». ${ }^{5}$

Et il remarque la différence entre «le droit ou quelque autre science humaine, où il n'y a ni vérité ni erreur», et où «la subtilité de l'esprit,... l'habilité de la parole... l'art de l'écrivain»... peuvent «faire apparaître ses raisons comme les meilleures». Mais dans un domaine comme celui des sciences naturelles, « où les conclusions sont vraies et nécessaires et n'ont rien à voir avec l'arbitraire humain», il peut arriver que «tel esprit médiocre... rencontre par hasard la Vérité», désarçonnant «mille Démosthène et mille Aristote» ${ }^{6}$. Car il s'agit ici d'un «mode d'entendement intensif », qui peut conduire, avec les mathématiques pures, à une «absolue certitude» - position qui lui sera reprochée par l'Eglise, comme signe d'orgueil et de mise en cause des seules vérités valables, les siennes.

Ainsi s'ouvre une nouvelle période de possibilités de connaissances: «Et qui voudrait poser des bornes au génie humain? Qui voudra assurer que l'on a déjà tout vu et su tout ce qu'il y a dans le monde de visible et d'intelligible? ${ }^{7}$

Ces avancées, un de ses amis qui essaie de le faire publier estime qu'elles représentent «le plus grand progrès en philosophie qui ait été fait depuis deux mille ans et qu'en frustrer le monde serait un méfait contre l'humanité ${ }^{8}$.

\section{Les risques du nouvel esprit}

Tout ceci, comme on sait, n'est pas allé sans risque. Giordano Bruno, un des premiers qui rompt avec l'idée d'un monde clos pour celle d'un monde infini, et qui propage l'idée de Copernic sur la rotation de la terre autour du Soleil, sera brûlé vif à Rome, condamné par le Saint-Office, en 1600. Galilée sera humilié et obligé de se rétracter, en 1633, à l'âge de soixante-dix ans. Ce n'est que très récemment (1992) que l'Eglise l'a réhabilité. Ces risques, les savants et philosophes de l'époque les ont affrontés selon leur personnalité propre.

Voilà un chanoine nommé Copernic, travaillant d'arrache-pied dans son lointain pays, et dont le nom devait passer à la postérité pour représenter le symbole même de la révolution scientifique. Copernic est un homme prudent. Il réserve ses découvertes à quelques amis et disciples sûrs. Il ne se décidera, sur leur pression, à publier son grand œuvre qu'à l'âge de soixante-dix ans, un peu avant sa mort. Cette œuvre, Sur les révolutions des corps célestes, Copernic l'adresse très respectueusement «A Sa Sainteté, le Pape Paul III».

J'ai pleinement conscience, ô Très Saint-Père, que certaines gens, dès qu'elles apprendront que, dans mon ouvrage sur les révolutions des corps célestes, j'attribue divers mouvements au globe terrestre, s'écriront aussitôt que pareille doctrine est absolument répréhensible.

Ainsi commence son texte. Il explique ensuite qu'il a voulu dédier son travail à un Pape connu pour son amour «de toutes les sciences et aussi des mathématiques » de sorte que «Votre autorité et Votre décision pourront me protéger de la

\footnotetext{
R. Zouckermann, Galilée, penseur libre, Paris, Editions de 1'Union Rationaliste, 1968, p. 43.

Op. cit., p. 95.

Op. cit., p. 267.

Op. cit., p. 170.
} 
dent des calomniateurs...». Et, pour finir, il déclare que ces travaux peuvent servir à améliorer le calendrier.

Les premiers savants sont donc des hommes seuls (avec quelques disciples et amis autour d'eux) qui doivent composer avec les dangers qu'ils courent en exprimant des opinions non admises par l'Eglise. Tel le médecin William Harvey, qui scandalise également avec sa découverte de la circulation du sang:

Il vaut beaucoup mieux chercher dans le silence de son logis à devenir savant pour son propre compte que de provoquer, en publiant prématurément des résultats qui vous ont coûté beaucoup de peine et de travail, des orages qui vous ôtent le calme et la paix de votre avenir'.

\section{La difficulté de convaincre}

Mais il ne suffit pas d'être arrivé à la vérité. Encore faut-il pouvoir en convaincre les autres. Et ce n'est pas toujours une mince affaire. Malgré les témoignages éclatants que Galilée apporte avec fierté, beaucoup refusent de changer d'opinion. Il y a les «obstinés», qui sont si fort enfermés dans leurs croyances qu'aucune preuve, qu'aucune démonstration ne pourra les faire changer d'opinion.

Votre Révérence m'a presque fait rire en disant qu'avec des observations si apparentes, on pourra convaincre les obstinés. Vous ne savez donc pas que pour convaincre ceux qui sont capables de raisonner et qui désirent connaître la vérité, les autres preuves étaient suffisantes; mais que, pour convaincre les obstinés, qui n'ont cure que des vains applaudissements du vulgaire, bête et stupide, le témoignage des Etoiles ne suffirait pas si elles descendaient à terre pour parler elles-mêmes ${ }^{10}$.

A quoi Galilée ajoute, faisant écho à Harvey:

Tachons seulement de savoir quelque chose pour nous-mêmes, restons-en à cette seule satisfaction.

Même entre «savants» partisans de la nouvelle manière de philosopher, l'accord n'est pas toujours aisé. Mersenne écrit à Galilée qui trouve ses formulations très obscures, et lui aurait fait répondre que «nous n'avons ici ni sphinx ni d'autres interprètes de mystères cachés », ce qui coupa court, semble-t-il, à leur échange ${ }^{11}$.

Quand à Kepler, le grand découvreur des lois célestes de l'époque, l'admiration qu'il témoigne pour Galilée n'est pas réciproque.

J'ai toujours tenu Kepler pour un esprit libre (peut-être trop libre) et subtil, mais mon mode de philosopher diffère beaucoup du sien. Il peut arriver qu'écrivant sur le même sujet, mais rarement et seulement sur ce qui regarde les mouvements célestes, nous nous soyons rencontrés sur quelques conceptions communes, et que nous ayons attribué la même cause vraie à un même effet réel: mais cela n'arrivera pas une fois sur cent ${ }^{12}$.

Cité par H. Kesten, Copernic et son temps, Paris, Calmann-Levy, 1951, p. 40.

R. Zouckermann, op. cit., p. 241.

Op. cit., p. 303.

Op. cit., p. 291. 
En effet, comme l'exprime Zouckermann, «son esprit encore médiéval contrastait avec celui de Galilée, qui annonçait déjà le XVIII ${ }^{\text {ème }}$ siècle. Ses livres sont alourdis d'un immense fatras de prévisions astrologiques, de grandes envolées mystiques et poétiques, d'élucubrations métaphysiques souvent cocasses ${ }^{13}$. Galilée:

On peut encore évoquer le rejet dogmatique par Descartes des résultats de

Tout ce qu'il dit de la vitesse des corps qui descendent dans le vide, etc... est bâti sans fondement; car il aurait dû auparavant déterminer ce que c'est que la pesanteur, et s'il en savait la vérité, il saurait qu'elle est nulle dans le vide ${ }^{14}$.

On peut remarquer ici que sont à l'œuvre des philosophies de la connaissance : Descartes, logicien avant tout, philosophe et mathématicien, cherche à «fonder » par le raisonnement les entités utilisées. C'est la raison pour laquelle les cartésiens seront contre la loi de Newton, qui fait appel à une gravitation qui apparaît ressortir de l'occultisme dont on veut se libérer. Dans les débuts d'une science mal implantée encore, ces philosophies s'affrontent dans des controverses où chacun pense détenir la vraie voie scientifique. Mais la science se bâtit peu à peu, aidée par une institution qui en s'installant va permettre que le travail de la preuve puisse se faire de façon plus systématique.

\section{La nécessité de rester entre soi}

Copernic, dans la présentation de son travail au Pape, fait remarquer que les idées d'un philosophe peuvent être très «éloignées de ce que juge la foule, puisqu'il a pour mission de rechercher la vérité en toutes choses ». Et rappelle que c'est la raison pour laquelle certaines écoles de pensée antiques, telle les pythagoriciens, transmettaient «la connaissance des mystères de la philosophie » oralement, et à quelques amis seulement et parents. Et ceci, non pas par «malveillance», mais pour se garder des moqueries et du mépris. Il écrit:

Je ne doute pas que les mathématiciens intelligents et érudits seront d'accord avec moi si, comme la philosophie l'exige avant tout, ils étudient non pas superficiellement mais à fond les preuves que j'apporte en cet ouvrage pour soutenir mon opinion, et s'ils veulent réfléchir.

William Gilbert, médecin et physicien, auteur du premier traité sur le magnétisme (De Magnete, 1600), conscient de l'impossibilité de convaincre tout le monde et de la nécessité d'une clôture de la cité scientifique, précise qu'il s'adresse seulement à ceux qui savent raisonner:

C'est pour vous seulement que j'écris, qui savez vraiment philosopher, hommes sans idées préconçues, qui cherchez la science non dans les livres seuls, mais dans les choses mêmes, que j’ai écrit ces principes de magnétisme, nés d'une nouvelle manière de philosopher ${ }^{15}$.

\footnotetext{
Op. cit., p. 325.

Lettre à Mersenne, 11 octobre 1638, cité par R. Zouckermann, p. 324.

15 De Magnete, 1600, cité par F. Russo, Nature et Méthode de l'Histoire des Sciences, Paris, Blanchard, 1983.
} 
On voit ainsi se profiler la nécessité de rester entre soi, de travailler et discuter avec ceux qui partagent la même philosophie, et les mêmes capacités pour ce type de connaissance. Car, dans les nouvelles sciences de la mécanique que fonde Galilée,

accepter la rigueur des démonstrations géométriques est une épreuve dangereuse pour qui ne sait pas bien les manier, car il n'y a pas de milieu entre le vrai et le faux: dans les démonstrations nécessaires, ou bien on arrive à la conclusion indubitable, ou bien on tombe dans des erreurs impardonnables.

Ainsi, ce «rester entre soi » demande la séparation d'avec ceux qui ne peuvent raisonner correctement en raison de leurs préjugés. «On ne peut rien attendre de bon de la fréquentation de tels gens, qui n'est pas seulement pénible mais dangereuse», estime Sagredo, un des personnages des dialogues de Galilée, qui écrit:

\begin{abstract}
... pour les conclusions qui ne peuvent venir à notre connaissance que par voie du raisonnement, je ne ferai pas beaucoup plus de cas du témoignage d'un grand nombre que de celui de quelques-uns, car je suis sûr que dans les questions difficiles, le nombre de ceux qui raisonnent bien est plus petit que celui de ceux qui raisonnent mal ${ }^{16}$.
\end{abstract}

\title{
La communauté scientifique
}

On sait que les académies des sciences seront fondées au courant du $17^{\text {ème }}$ siècle pour favoriser ces regroupements entre personnes sachant manier l'esprit de rigueur. La communauté scientifique va permettre de contrôler au plus près le raisonnement, les expériences, et empêcher, sans pouvoir toutefois les supprimer entièrement, les dérives. C'est un monde vivant, où la recherche ne se fait pas selon une simple logique, mais où imagination, observation, calcul, s'entremêlent constamment, et pas toujours dans le même ordre.

Descartes s'interrogeant sur la bonne méthode à suivre, celle qui pourra conduire à des certitudes, notait: « les causes de désaccord proviennent d'une part, d'un manque de méthode, d'autre part, du fait que l'on ne considère pas les mêmes choses ».

Peu à peu, au sein de la communauté scientifique, se précise la définition des «objets » dans les sciences exactes - phénomènes, appareillages, instruments, formules mathématiques - ce qui permet précisément aux mathématiciens, aux physiciens, aux biologistes, de «considérer les mêmes choses».

\section{Controverses}

Ce travail d'identification est primordial et loin d'être évident. D'où l'existence de controverses, spécifiques de ce type de travail, car qui dit controverse, dit aussi la croyance en l'existence d'une réponse, en la possibilité d'arriver à une conclusion. Je ne prendrai ici qu'un exemple, qui apparaît dans un livre anti-sokalien ${ }^{17}$, donné par S.J. Gould, repris par I. Stengers. Ces deux scientifiques sont sympathisants des post-modernes, mais pour avoir pratiqué l'un et l'autre la science, ils tiennent à rappeler à leurs amis qu'elle est capable de donner des résul-

\footnotetext{
$16 \quad$ R. Zouckermann, op. cit., p. 147.

17 B. Jurdant, Impostures scientifiques, Paris/Nice, La découverte/Alliages, 1998, p. 279.
} 
tats précis. Il s'agit de la façon dont des géologues sont parvenus à résoudre des points controversés touchant la datation de la Terre.

(Les géologues) ne peuvent vivre leurs conflits sur un mode tranquille, cynique ou résigné. Ils sont contraints à accumuler de nouvelles données, à construire de nouveaux arguments, à prendre en compte les divergences, à multiplier toujours plus les éléments d'une situation afin de créer les conditions d'une convergence possible. Ce que les géologues cherchaient, cherchaient passionnément, n'était pas seulement la victoire sur leurs adversaires, c'était ce que Gould appelle une «douce victoire».

Ces résultats qui dénouent la controverse, les «vaincus peuvent s'(en) réjouir autant que les vainqueurs» et tous peuvent «célébrer» ensemble les «triomphes de la connaissance humaine», qui permettront de poser de nouvelles questions. Koyré parlait de «dialogue avec la nature». Et l'on nomme «expérience cruciale», l'expérience capable de fournir une réponse sans ambiguiité à une controverse.

\section{La confiance}

Lettre de Kepler à Galilée (19 avril 1610) : «On me regardera peut-être comme téméraire de vous croire si facilement, sans le soutien d'aucune observation personnelle. Mais qui ne croirait pas un si savant mathématicien, dont le style même garantit la droiture du jugement»..

J'ai souligné une partie de la phrase, qui me semble importante pour illustrer le rôle fondamental que joue la confiance dans le travail scientifique.

On ne peut pas refaire tous les calculs, et toutes les expériences. On va donc faire confiance à ceux qui ont fait ces calculs, ou pratiqué ces expériences, dans la mesure où il est acquis qu'ils connaissent les règles de l'art et sont absolument honnêtes. Dans une certaine mesure, les scientifiques sont interchangeables par rapport à l'objet étudié et découvert, étant donné que, si le résultat est important, il va faire l'objet de vérifications poussées.

Cette notion de confiance est essentielle: dans un monde où la spécialisation est poussée à outrance, il faut que l'expert se montre digne de cette confiance, ce qui n'est pas toujours le cas. On rencontre là un des problèmes majeurs de notre société.

\section{Emerveillements}

Dans les débuts de la science, on trouvait déjà merveilleux de découvrir «la bonne manière de philosopher», des résultats comme la circulation du sang, ou encore l'emploi de lunettes astronomiques pour mieux regarder les planètes. On sait le succès, au cours du $18^{\text {ème }}$ siècle, de ces «cabinets de curiosité » ou « de physique », où l'on pouvait, par exemple, éprouver sur soi-même les effets d'un courant électrique.

Le développement de la science n'a fait que renforcer, chez les scientifiques des sciences exactes, ce sentiment d'émerveillement, cependant que, à mesure qu'elles devenaient plus spécialisées et ésotériques, le monde non scientifique n'en pouvait juger que les retombées techniques. L'émerveillement ne porte plus seulement sur les phénomènes étonnants découverts ou sur le bon accord entre une formule mathématique et des données expérimentales, mais sur le fait que des 
résultats dans des domaines locaux peuvent s'étendre à d'autres domaines, de façon absolument pas prévue au départ. C'est une architecture impressionnante qui a été engendrée, dont la cohérence est souvent allée avec une simplification inimaginable a priori. Ainsi, les équations de Maxwell, obtenues de façon plutôt confuse par lui-même, ont pu par la suite être réécrites de façon simple, grâce à un nouvel outil mathématique, le calcul vectoriel. Cette simplicité de l'architecture obtenue permet à Feynman, dans un des volumes de son traité d'enseignement de la physique ${ }^{18}$, de mettre dans une seule page (p.310) les huit équations qui résument la physique classique: quatre sont celles de Maxwell, auxquelles se rajoutent (toujours en électromagnétisme) une loi de conservation de la charge qui se déduit, de fait, des équations précédentes, et une loi de force, et, en dehors de l'électromagnétisme, la loi du mouvement (de Newton, modifiée par Einstein) et celle de la gravitation. «Personne n'a jamais trouvé une expérience qui soit en désaccord avec elles».

\begin{abstract}
Nous avons donc en une seule petite table toutes les lois fondamentales de la physique classique... Nous vivons un grand moment... Nous avons travaillé dur pour atteindre ce point $^{19}$.
\end{abstract}

Dans un article publié en 1960, le physicien E. P. Wigner s'interroge sur «l'efficacité déraisonnable des mathématiques dans les sciences naturelles ${ }^{20}$. Il remarque, entre autres exemples, que la loi de Newton concernant l'attraction des corps à travers les phénomènes de gravitation était valable avec une erreur de $4 \%$ quand il l'a établie. Cela n'aurait pu être qu'une quantification pratique d'un phénomène. Or, après plus de deux siècles de travaux intenses, après un développement sans précédent de la connaissance en physique, en profondeur comme en extension des domaines, la loi s'avère actuellement exacte avec une erreur de moins d'un dix millième de pourcentage. Au cours de son exposé, Wigner utilisera le terme de «miracle» une dizaine de fois. Sa conclusion: «Le miracle de la justesse du langage mathématique pour la formulation des lois de la physique est un don merveilleux que nous ne comprenons ni méritons ». Il rejoint ainsi, entre autres, Einstein qui disait que le plus incompréhensible était que l'univers (physique) est compréhensible.

Donnons un dernier exemple. Jean Perrin, dans un livre classique ${ }^{21}$, s'efforce de démontrer la réalité de l'existence des atomes, en approchant le phénomène atomique, à travers le nombre d'Avogadro, de plusieurs façons. Il calcule ce nombre en utilisant des théories physiques différentes, concernant «des phénomènes de prime abord aussi complètement indépendants que la viscosité des gaz, le mouvement brownien, le bleu du ciel, le spectre du corps noir ou la radioactivité ». Il obtient de la sorte 13 mesures, qui sont étonnamment proches: entre 60 et $75 \cdot 10^{-22}$.

\footnotetext{
R. Feynman, Electromagnétisme 1, Paris, InterEditions, 1979.

Op. cit., p. 315.

20 E. P.Wigner, The Unreasonable Effectiveness of Mathematics in the Natural Sciences, in «Communications on Pure and Applied Mathematics », 13, 1960, pp. 1-14.

21 J. Perrin, Les atomes, Paris, Gallimard, 1970, pp. 274-275.
} 
On est saisi d'admiration devant le miracle de concordances aussi précises à partir de phénomènes si différents... puisque les nombres ainsi définis sans ambiguïté par tant de méthodes coïncident, cela donne à la réalité moléculaire une vraisemblance bien voisine de la certitude.

\section{Esthétique}

Un aspect proche de cet émerveillement, et le plus souvent peu compréhensible pour l'étranger à cette culture, est l'aspect esthétique de la construction. Le beau n'est pas le but premier de la science, et pourtant, ces concordances, ces cohérences inattendues, sont sources de plaisirs esthétiques intenses, au point que certains vont guider leur recherche à partir de l'esthétisme des formules. On parle de «beaux théorèmes», de «belles démonstrations», de «belles expériences». Henri Poincaré:

Les combinaisons utiles, ce sont précisément les plus belles, je veux dire celles qui peuvent le mieux charmer cette sensibilité spéciale que les mathématiciens connaissent ${ }^{22}$.

L'homme de science n'étudie point la nature parce que c'est utile; il l'étudie parce qu'il s'y plaît, et il s'y plaît parce que c'est beau ${ }^{23}$.

Le cas de l'équation de Dirac est un exemple bien connu. Dirac a ajouté un terme dans l'équation qui porte son nom par souci de symétrie, par une intuition que cette symétrie avait un sens dans la description des particules élémentaires. Cette symétrie fournissait ainsi l'hypothèse d'une particule correspondant à l'électron, mais pourvue, elle, d'une charge positive. Cette hypothèse fut vérifiée, et le positon vint prendre sa place dans la collection des particules élémentaires.

C'est au nom de l'esthétique que le physicien J. Treiner ${ }^{24}$, dans la dispute autour de l'affaire Sokal, proteste sur les déformations de la réalité de la physique par certains sociologues ou philosophes:

Cela n'aurait pas d'importance? Réaffirmons que cela en a. Il en va des théories physiques comme d'un blues de Tom Waits ou comme de certaines parties d'échecs. Il y en a de belles, de très belles, c'est de la vraie beauté qui prend au ventre... C'est toute une construction... qui se trouve bafouée par la désinvolture du monsieur ${ }^{25}$. Un effet de manche, et vlan! Le jeu d'échecs est par terre, la poterie en pièces, la beauté déchirée.

Le mathématicien Laurent Schwartz est connu à la fois pour ses contributions majeures en analyse mathématique et pour ses participations, en tant qu'intellectuel, à divers combats de justice dans la société. C'est au cours de «la plus belle nuit de sa vie» qu'il crée les «distributions», êtres mathématiques qui permettaient de remettre sur pied, au niveau de la rigueur mathématique, un type de «fonctions» qu'avait introduites le physicien Dirac, déjà nommé ci-dessus.

22 H. Poincaré, Science et méthode, Paris, 1908, p. 58, cité par A. I. Miller, « Diogène », n 177, janv.mars 1997.

23 H. Poincaré, La valeur de la science, cit. id.

24 J. Treiner, Sokal-Bricmont: non, ce n'est pas la guerre, «Le Monde», 11.10.97. Cité par J. Feldman, $L$ ' «Affaire SOKAL»: Un épisode de la méconnaissance entre cultures, in "L'Année Sociologique», 49, 1999, pp. 245-270.

25 Il s'agit ici, nommément, de Michel Serres. 
Celles-ci s'avéraient utiles, mais étaient «totalement dénué(es) de sens » aux yeux des mathématiciens «écœurés». Dans son autobiographie ${ }^{26}$, Laurent Schwartz a un chapitre intitulé «la révélation des mathématiques». Il y raconte comment il a été «séduit»par la géométrie, dans la dernière année de sa scolarité au lycée. Toutes les parties de son livre concernant les mathématiques sont un hommage vibrant à leur beauté. Il y parle de «magnifique architecture » et explique qu'elles représentent pour lui un «château intérieur».

Et puisque cette beauté là est si difficile à comprendre pour le profane, le physicien Bernard Diu en donne un équivalent: il agrémente son livre Les atomes existent-ils vraiment? ${ }^{27}$ de nombreuses poésies. Il s'en explique dans un avertissement: «J'ai ressenti le besoin que la physique, si belle en ce monde si dur, fût accompagnée et entourée d'un essaim d'autres beautés, plus fragiles et plus labiles certes, mais sans doute plus accessibles aussi ».

\section{Les grands noms}

Dans cette foule immense de ceux qui ont participé à la construction de ces architectures, la presque totalité restera anonyme. On fera ressortir pourtant les noms de quelques «grands savants», dont on conserve avec piété la mémoire. Ce sont ceux dont on considère qu'ils ont apporté quelques pierres particulièrement neuves, et importantes, à l'édifice.

On ne garde ainsi, de leurs travaux, que ceux qui se sont avérés participer à la construction de l'édifice. On ne retiendra des nombreux travaux de Kepler que trois de ses lois, dites «lois de Kepler». Certains vont ainsi avoir la chance de voir leur nom attaché à leur découverte: effet Mossbauer, constante de Planck, équation de Schrödinger, principe de Fermat, loi de Boyle, coordonnées cartésiennes, pour citer quelques exemples au hasard. Parfois, ce sont des unités de mesure qu' on baptisera du nom de celui auquel on veut rendre hommage, tel le «becquerel », qui mesure l'activité d'une source radioactive, ou encore le «Pascal, unité de pression équivalant à la pression exercée par une force de Newton sur une surface plane de 1 mètre carré ». Dans cette définition, on rappelle ainsi les travaux du premier de ces savants sur la pression atmosphérique, et ceux du second sur la force de gravitation.

Ainsi revient le sujet qui avait été refoulé. La science n'est en effet pas pure logique, objectivité totale. On sait que les démonstrations de mathématiques ne peuvent éviter le langage commun. En principe, il serait possible de le supprimer totalement et de faire d'un texte mathématique un texte uniquement logique. Or, le résultat s'avère impossible à lire: la logique n'est pas la mathématique qui, alors même qu'elle s'occupe d'objets parfaitement définis, a besoin d'une certaine dose d'aération, d'un peu de support intuitif. De la même façon, je verrais dans le besoin de mentionner et d'admirer les auteurs des découvertes un besoin d' «humaniser» la science, en tentant de mieux faire connaître ceux qui la font. Et ceci, peut-être, d'autant plus que leurs découvertes sont plus difficiles à comprendre. Chaplin disait, parait-il, à Einstein: «on m'aime parce que tout le monde me comprend, et vous, parce que personne ne vous comprend».

\footnotetext{
26 L. Schwartz, Un mathématicien aux prises avec le siècle, Paris, Ed. Odile Jacob, 1997.

27 B. Diu, Les atomes existent-ils vraiment? Paris, Ed. Odile Jacob, 1997, p. 19.
} 
Aujourd'hui, ce besoin de connaître ces personnalités hors pair passe évidemment par les médias, et par le prestige des prix proposés par l'ingénieur Alfred Nobel, à la fin du $19^{\text {ème }}$ siècle. Quelques personnages particulièrement «médiatiques » sont ainsi propulsés sur le devant de la scène, dont on voudra connaître tout, la vie, les sentiments, les engagements politiques, etc. Ces effets de loupe font apparaître encore plus gris le scientifique moyen, qui travaille dans une cité scientifique devenue gigantesque, et pour une science désormais soumise à la défiance. Cette ambiance n'est sans doute pas tout à fait étrangère aux frustrations qui font que certains scientifiques éprouvent eux aussi, le besoin de «dire je». disait:

Le physicien Pierre Auger (mort en 1993), qui a découvert «l'effet Auger»,

«L'honneur suprême, c'est de perdre la majuscule de son nom. Quand je vois, écrit sur un tableau de commande, au-dessous d'un bouton, 'auger', je me dis que j'ai bien de la reconnaissance envers cet effet dont j'ai l'honneur de porter le nom ${ }^{28}$.

\title{
II. LES POSTURES DU CHERCHEUR DANS LES SCIENCES DE L'HOMME
}

\author{
I have felt an increasing disconfort at the \\ distance between the rigourous objectivity of \\ myself as a scientist and the almost mystical \\ subjectivity of myself as therapist
}

Carl R. Rogers

\section{L'institutionnalisation des sciences sociales}

L'histoire des sciences sociales est tout autre puisqu'elle commence dans un environnement où la science est acceptée et encouragée. C'est même en raison des succès obtenus par les sciences exactes que vont être institutionnalisées à leur tour les sciences de l'homme. Leur principal défi sera de tenter d'adapter au cas de l'étude des réalité humaines et sociales les manières de faire qui ont si bien réussi dans les sciences exactes. Le grand débat qui sous-tend, depuis deux siècles, le travail considérable, par le nombre comme par les idées, fait dans les sciences de l'homme, porte sur les possibilités de cette adaptation. Leur grand souci est de faire reconnaître leur légitimité en tant que science. Pour cela, on affaiblira le plus souvent la définition de la science, de sorte qu'il soit possible de s'en réclamer.

Tous ceux qui pensent la scientificité, tels Bacon, Condorcet, Mill, Comte, se sont exprimés sur cette question. Pourtant, quelques grands ancêtres de la pensée sociologique, tels Montesquieu et Tocqueville - dont on se plait souvent à souligner la pertinence toujours actuelle de l'analyse qu'il a faite de la démocratie moderne - ne s'en sont guère inquiétés, peut-être parce l'un et l'autre se sentent maîtriser leur pensée logique. Ils s'attachent simplement à réfléchir du mieux qu'ils peuvent sur les problèmes de la société.

28 Cité par J.-F. Augereau, «Le Monde», 29 mai 1999. 
Une entreprise intéressante est celle à laquelle se sont livrés, dans les années soixante, Berelson et Steiner ${ }^{29}$. Il ont voulu rassembler les résultats auxquels seraient arrivées les sciences de l'homme après un siècle d'existence. L'ouvrage contient plus de 700 pages et porte sur 14 grands thèmes («perception», «famille $», \ldots)$. Chaque chapitre donne des définitions et des résultats, le plus souvent présentés sous la forme de "propositions», par exemple (pris au hasard): «Le moins assuré le groupe est quant à ses normes, le moins de contrôle il exerce sur ses membres». A ma connaissance, cet effort n'a pas été continué. $\mathrm{Au}$ contraire, il procure un certain malaise par ce qu'on dénommera son «positivisme», autrement dit sa volonté de résumer ainsi des situations complexes. Notons que sont préférés actuellement les dictionnaires faisant le point sur les notions utilisées dans divers champs de la recherche.

L'importance des dissensions sur le jugement d'ouvrages reconnus comme scientifiques, à travers les thèses de doctorat, par exemple, est sans commune mesure avec ce qui peut se produire dans les sciences exactes. On ne fera ici que citer le dernier «scandale» qui a secoué la cité des sociologues, l'acceptation d'une thèse de sociologie soutenue par une astrologue.

Une épistémologie éclatée, des méthodes diverses, dont on n'a pas encore terminé, à mon avis, le recensement, voilà ce qui va caractériser les sciences sociales, prises entre philosophie, littérature, journalisme, et science proprement dite. La complexité ontologique de la réalité sociale et humaine appelle à différentes approches. Dans la mesure où elles se juxtaposent souvent sans pouvoir donner lieu à de véritables discussions, la dénomination de cité scientifique, plutôt que de communauté scientifique, me semble mieux convenir $\mathrm{ici}^{30}$. Bourdieu, dont on connaît la tendance polémiste, disait: «je n'ai que des ennemis, je n'ai pas d'adversaires $»^{31}$.

Une comparaison avec certains thèmes de la section précédente pourrait être menée. Par exemple, il serait intéressant de voir où se situe les plaisirs du travail (belle écriture, belle pensée, inventivité, capacité de «déconstruction», parfois aussi, séduction), de nature pourtant différente. L'engagement dans la cité est par contre plus présent, alors que le physicien peut vivre dans une disjonction totale son éthique interne, celle qui consiste à faire du bon travail, et son éthique externe, celle du citoyen.

Pour ce qui est des «grands noms», se marque aussi une différence notoire avec le cas des sciences exactes. Comme la littérature et la philosophie, la sociologie possède ses classiques. Ce sont les auteurs qui ont apporté une pensée sociologique jugée importante qui sont retenus. Or, une «pensée» reste, même si elle influence beaucoup, d'un caractère essentiellement étendu, complexe, personnel. Elle ne se laisse pas réduire à quelques résultats précis. C'est pourquoi on a toujours intérêt à relire Platon, Spinoza, Auguste Comte, Weber, Durkheim, Aron dans le texte, plutôt que de s'en tenir à leurs commentateurs.

29 B. Berelson \& G. A. Steiner, Human Behavior ; An Inventory of Scientific Findings, New York, Harcourt, 1964.

30 J. Feldman, Deux fois deux axiomes sur les différences entre les sciences exactes et les sciences sociales in «Revue Européenne des sciences sociales», n 120, 2001, pp. 191-205.

31 Dans un entretien sur France-Culture avec l'historien Roger Chartier. 
Dans cette aventure d'exploration de champs de connaissance nouveaux, je vais tenter de repérer diverses attitudes qui sont employées. J'utilise le terme «posture» pour en faire percevoir le côté volontariste, conscient, et relativement difficile à tenir.

\title{
La posture de distanciation et d'objectivité
}

La première règle d'or donnée aux étudiants est une règle de distanciation, de rupture avec le sens commun, qui est supposée d'autant plus nécessaire que nous appartenons à cette société que nous désirons étudier.

Emile Durkheim, en 1895, explicite cette règle dans Les règles de la méthode sociologique ${ }^{32}$.Relisons-le:

\begin{abstract}
S'il existe une science des sociétés, il faut bien s'attendre à ce qu'elle ne consiste pas dans une simple paraphrase des préjugés traditionnels, mais nous fasse voir les choses autrement qu'elles n'apparaissent au vulgaire; car l'objet de toute science est de faire des découvertes et toute découverte déconcerte plus ou moins les opinions reçues. A moins donc qu'on ne prête au sens commun, en sociologie, une autorité qu'il n'a plus depuis longtemps dans les autres sciences - et on ne voit pas d'où elle pourrait lui venir - il faut que le savant prenne résolument son parti de ne pas se laisser intimider par les résultats auxquels aboutissent ses recherches, si elles ont été méthodiquement conduites.
\end{abstract}

Dès la deuxième phrase de la préface s'affirme ainsi le souci de se distinguer $\mathrm{du}$ «vulgaire», ce qui permet de mieux revendiquer une reconnaissance institutionnelle, but auquel s'était attaché Durkheim. En effet, dans la préface de la deuxième édition, Durkheim se félicite de ce que, «en dépit des oppositions, la cause de la sociologie objective, spécifique et méthodique a gagné du terrain sans interruption. La fondation de l'Année sociologique a certainement été pour beaucoup dans ce résultat ».

Mais cette règle de distanciation n'est pas facile à appliquer:

\begin{abstract}
Nous sommes encore trop accoutumés à trancher toutes ces questions d'après les suggestions du sens commun pour que nous puissions facilement le tenir à distance des discussions sociologiques. Alors que nous nous en croyons affranchis, il nous impose ses jugements sans que nous y prenions garde. Il n'y a qu'une longue et spéciale pratique qui puisse prévenir de pareilles défaillances. Voilà ce que nous demandons au lecteur de bien vouloir ne pas perdre de vue. Qu'il ait toujours présent à l'esprit que les manières de penser auxquelles il est le plus fait sont plutôt contraires que favorables à l'étude scientifique des phénomènes sociaux et, par conséquent, qu'il se mette en garde contre ses premières impressions.
\end{abstract}

Voilà donc une attitude de l'esprit qui constitue une rupture avec nos habitudes. Durkheim précise ici sa philosophie: «Notre premier objectif... est d'étendre à la conduite humaine le rationalisme scientifique», la découverte des causes permettant dans un deuxième temps de déterminer des règles d'action.

Cette règle de distanciation conduit à une nouvelle division de l'esprit humain, introduite une première fois dans la vision du monde extérieur par les sciences exactes. «Les faits sociaux sont comme des choses », dit Durkheim.

32 E. Durkheim, Les règles de la méthode sociologique, Paris, PUF, 1968 (1895). 
La difficulté de cette séparation, Durkheim l'aperçoit aussi par rapport à la morale. La première objection qu'il va contrer, c'est celle qui consiste à lui reprocher «d'absoudre le crime, sous prétexte que nous en faisons un phénomène de sociologie normale». De fait, il y a là un choix philosophique si peu évident qu'il continue à faire problème. On reprochera souvent au sociologue ou, plus généralement, à la personne qui examine un acte avec distance, que l'explication qu'elle en propose empêche son jugement moral. Cette discussion habite les tribunaux ordinaires, où il s'agit de décider du degré de responsabilité d'une personne dont les avocats vont mettre en avant les circonstances malheureuses de sa vie, mais aussi des événements historiques lourds ${ }^{33}$.

J. Amery, décrivant son expérience des camps nazis, rejette violemment toute objectivité.

L'objectivité que l'on me réclame dans le débat avec les bourreaux, avec ceux qui les aidaient, avec les autres qui les regardaient en silence, me semble logiquement absurde. Le méfait n'a en tant que méfait aucun caractère objectif. Le massacre, la torture, la mutilation... ne sont, objectivement, que des enchaînements d'événements physiques, descriptibles dans la langue formalisée des sciences exactes; ce sont des faits à l'intérieur d'un système physique, non des actes inscrits dans un système moral... J'étais et je suis le seul à posséder la vérité morale des coups qui aujourd'hui encore résonnent dans mon crâne... mes ressentiments sont là pour que le crime devienne une réalité morale aux yeux du criminel lui-même, pour que le malfaiteur soit impliqué dans la vérité de son forfait.

On m'a infligé une blessure. Tout ce que je dois faire c'est la désinfecter et la bander, et non pas réfléchir à la raison pour laquelle le bourreau a levé sa hache, et finir sans doute par le disculper en découvrant cette raison ${ }^{34}$.

Le linguiste reconnu Tzvetan Todorov quitte un beau jour les sciences sociales où il considère que le souci éthique n'est pas assez fort, pour rejoindre la tribu des essayistes, et abandonner les prétentions scientifiques.

Quand je suis devenu conscient de cette séparation, j'ai commencé à éprouver une insatisfaction - grandissante - des dites sciences humaines et sociales... telles qu'on les pratique en général aujourd'hui. La rupture entre vivre et dire, entre faits et valeurs me semble, pour elles spécifiquement, néfaste (la rupture, non la distinction: on peut aussi distinguer et relier). C'est là, en effet, que je situe la différence la plus intéressante entre les sciences humaines et les autres (celles de la nature)... Pour moi, la différence dans la matière étudiée (humain/non humain) en entraîne une autre, capitale, dans le rapport qui s'établit entre le savant et son objet... Ici, une pensée qui ne se nourrit pas de l'expérience personnelle du savant dégénère vite en scolastique... Et c'est ainsi que, aux sciences humaines et sociales, j'en suis venu à préférer l'essai moral et politique ${ }^{35}$.

Pourtant, pour beaucoup, la découverte de l'approche sociologique peut constituer un moment important de la vie de l'esprit, comme une conversion,

33 C'est ainsi que le cinéaste et écrivain C. Lanzmann refuse de faire de la Shoah un événement ordinaire, qu'on pourrait expliquer. Toute tentative d'explication lui apparaît comme ratant l'essentiel du phénomène.

34 J. Amery, Par delà le crime et le châtiment, essai pour surmonter l'insupportable, Actes Sud, 1995, pp. 122-123 et p. 153.

35 T. Todorov, Nous et les Autres. La Réflexion française sur la diversité humaine, Paris, Seuil, 1989, p. 10. 
conversion dans la posture, prise de conscience, prise de distance par rapport à ce qui paraissait évident, que l'on n'avait en conséquence pas l'idée d'interroger, et qui s'avère pris dans des sens, dans des logiques, dans des explications relatives aux sociétés. Les connaissances sociales ne sont pas sans effets sur les personnes.

Ainsi cette jeune femme qui tient un petit hôtel du sud du massif central, où je m'installe pour une semaine dans une petite chambre. Je lui ai demandé une table, pour pouvoir y installer ma machine à écrire. Non, je ne suis pas écrivain, mais sociologue. Je pense que, comme beaucoup d'autres, elle ne saura pas ce que c'est. Je me trompe, elle a fait une année d'étude de sociologie à Annecy, avant de venir s'établir ici. Cette étude l'a bouleversée.

On sait aussi que cette mise en logique sociale peut être source, éventuellement pénible, de désenchantement: ainsi ce photographe amateur qui, après avoir appris de Bourdieu qu'il pratiquait un «art moyen» faute d'avoir eu accès à des arts plus nobles, s'en est dégoûté.

La prise de conscience de l'existence, à travers les récits ethnographiques, de mœurs très différentes de celles auxquelles on a été accoutumé, peut produire également un choc d'importance. Telle cette adolescente à qui, au lycée, on a fait lire les livres de Paul-Emile Victor «Banquise» et «Boréal», sur la vie de ceux que l'on appelait alors les esquimaux. En particulier, vont la choquer la promiscuité mais aussi l'utilisation importante de l'urine, un élément supposé sale chez nous. Or, voilà que ceci est présenté comme normal, là-bas. Le choc réside en ceci: l'adolescente prend conscience que, si elle était née sous d'autres cieux, elle serait très différente. Des pans entiers de sa personnalité, ceux qui concernent le dégoût, l'intimité, par exemple, seraient changés. Cela apparaît comme une menace sur son identité. Il est probable que cette menace est du même ordre que celle qui affecte certains jeunes de nos banlieues dites difficiles, que l'on dit «sans repères ».

Certains refusent, on l'a vu, cette posture de distanciation dans sa propre société: soit que, philosophes plus généralistes, ils s'en méfient, soit que, grands blessés de la vie, leur priorité est le cri de colère. Il y a plus : la sociologie exige un certain type d'esprit d'abstraction, ou l'acceptation d'un certain degré de séparation dans la personnalité, que les opposants estimeront peut-être à tendance schizophrénique. La psychologie est plus acceptée: c'est que nous sommes tous, plus ou moins bons, psychologues. Il y a là un concret que l'on pratique constamment, avec plus ou moins de problèmes, avec les autres. La démarche psychanalytique, par contre, demande elle aussi un certain type de conversion: s'arrêter sur ce qui apparaît comme des détails de la vie sans importance pour leur chercher des significations; rompre avec les habitudes de la pensée pour suivre systématiquement l'illogisme des associations d'idées et aller ainsi au hasard - du moins, c'est ce qui semble-explorer son monde intérieur; surtout, accepter de prendre conscience de l'existence de résistances, d'aveuglements, de dénis, de zones entières de nonsavoir.

Ces phénomènes touchent aussi la perception du social. Weber a introduit la notion d'idéal-type en tant que modèle d'une réalité toujours complexe dont on extrait des traits que l'on juge essentiels. Il n'est pas facile de démontrer cette «essentialité ». Certaines réalités sociales nous affectent particulièrement, et nous pouvons les ressentir de façon forte, alors que d'autres ne les ressentiront guère ou pas du tout, et seront portés à minimiser leurs manifestations, voire, à les nier. 
S'il est bien des exemples dans les sciences exactes de refus, au départ, de nouvelles idées explicatives, parce qu'elles heurtent profondément certaines habitudes de pensée, dans le cas des sciences humaines, ce n'est pas seulement d'idées intellectuelles dont il s'agit, mais de perceptions à la fois intellectuelles et affectives, qui peuvent être imprimées dans les profondeurs de la personnalité.

Un exemple me paraît être un véritable cas d'école: ce fait social majeur que représente le genre, dans ses rapports avec la cité des sociologues, où chacun est atteint par la prégnance des inculcations faites dès la prime enfance. Il est symptomatique que les deux pères fondateurs de la sociologie aient eu au sujet du féminisme des positions bien tranchées et opposées: Durkheim s'est élevé contre le féminisme, au nom du destin naturel de la femme (sic!). Weber, dont la femme était féministe, s'il n'a guère abordé le thème dans ses travaux, a soutenu son combat.

P. Ansart a mené une réflexion sur le thème: «Toute connaissance du social est-elle idéologique?» ${ }^{36} \mathrm{Il}$ propose d'écarter «l'idée messianique» d'une science positive, qui pourrait arriver à se débarrasser, à la longue, des idéologies imprégnant la pensée sociologique. A travers divers exemples - Marx, Durkheim, Weber... -, il montre que ces penseurs ont à la fois rempli le programme de la sociologie qui consiste en une déconstruction de certaines prénotions et /ou idéologies sociales, mais ont également subi certaines de celles qui étaient de leur temps. Et c'est en raison même de leur implication dans ces dernières qu'ils ont pu faire œuvre novatrice. Aussi, pour lui, il faut abandonner l'espoir de départager le vrai et le faux, comme cela peut se faire dans les sciences exactes et accepter l'ambiguïté essentielle de la sociologie.

Le cas des statistiques sociales peut-il être évoqué comme exemple de l'objectivité possible de la sociologie? Elles se bornent en effet à l'observation la plus exacte possible de réalités, dont les catégories fondamentales sont cependant fournies par la société. Tous ceux qui se sont essayés à des comparaisons internationales sur base de statistiques nationales ont pu faire l'expérience des variations, d'un pays à l'autre, de ces catégories. Mais il y a là des plages d'objectivation possibles, surtout si l'on sait à peu près en reconnaître les limites.

\section{Les postures de proximité}

Je vais ici examiner de quelles façons, diverses, des chercheurs des sciences de l'homme ont fait intervenir la subjectivité du chercheur, de façon générale ou en partant de leur propre expérience.

\section{La compréhension weberienne}

Comme on le sait, le courant allemand va opposer les sciences de la nature et les sciences humaines, dites aussi «sciences de l'esprit»: avoir affaire à un être humain est fondamentalement différent d'avoir affaire à une chose, et peut être de fait - un avantage, du point de vue de la profondeur de la connaissance. En effet, si l'on manque, dans les secondes, des possibilités de manipulation et d'ex-

36 P. Ansart, Toute connaissance du social est-elle idéologique?, in J. Duvignaud, Sociologie de la connaissance, Paris, Payot, 1979. 
périmentation que possèdent les premières, celles-ci ont un nouvel atout: la possibilité de comprendre les êtres humains, parce que le chercheur et son «objet d'études » relèvent tous les deux de la condition humaine. Voilà ce qui distingue et, d'une certaine manière, favorise les nouvelles sciences de l'homme.

Weber examine avec attention cette notion de compréhension, car il n'admet que difficilement d'avoir à faire le deuil de la scientificité. Cette scientificité dont il reconnaît qu'elle ne sera jamais aussi complète que dans les sciences de la nature - les sciences de l'homme sont destinées à toujours «rester jeunes »-il la définit dans la possibilité de déterminer les causes qui relient entre eux les phénomènes. Il propose alors un dégradé de types de compréhension des actions de l'être humain. Celui dont l'action est parfaitement rationnelle, c'est-à-dire adaptée à une intention bien spécifiée, est aussi compréhensible qu'une explication dans les sciences de la nature. Celui dont l'action est la moins compréhensible, la plus éloignée d'une explication scientifique, parce que la moins partageable, est celle suscitée par une affectivité irrationnelle.

Ce type de compréhension demeure donc dans une posture relativement distante. En somme, Weber ne peut s'identifier aux personnes étudiées que dans la mesure où elles sont rationnelles. Depuis, diverses approches de la connaissance humaine vont s'attacher à apprivoiser un peu le vaste monde de l' «irrationalité », en osant faire intervenir la propre personne du chercheur, hors de sa propre rationalité.

\section{L'histoire et l'historien, vus par H.-I. Marrou}

En 1954, l'historien H.-I. Marrou ${ }^{37}$ affirme que «l'histoire est inséparable de l'historien ${ }^{38}$, et que «l'histoire est une aventure spirituelle où la personnalité de l'historien s'engage tout entière ${ }^{39}$. Même s'il met en garde de ne pas «trop appuyer sur la pédale existentielle», il tient à affirmer que, en histoire, «l'objectivité n'est pas le critère suprême ${ }^{40}$, la vérité étant au moins aussi importante, et ne s'y ramenant pas nécessairement. Contrairement à Durkheim, il pense qu' «il faut prendre son point de départ dans la connaissance dite vulgaire ${ }^{41}$ - position qui, par ailleurs, rejoint celle des physiciens Sokal et Bricmont - même s'il finit tout de même par retrouver le premier par le besoin de se séparer de ses préjugés. Seulement, cela sera fait peu à peu, au cours du travail, et pour mieux rencontrer «autrui »:

Toute l'expérience historique se présente, pour le chercheur, comme une ascèse où, au contact des documents, il apprend peu à peu à se dépouiller de ses préjugés, de ses habitudes mentales, de sa forme trop particulière d'humanité... pour se rendre capable de comprendre, de rencontrer autrui ${ }^{42}$.

\footnotetext{
H.-I. Marrou, De la connaissance historique, Paris Seuil, 1975 (1954).

Op. cit., p. 2.

Op. cit., p. 196.

Op. cit., p. 48.

Op. cit., p. 81.

Op. cit., p. 145.
} 
La véritable rigueur consiste à reconnaître cet engagement du chercheur, dont «la richesse de connaissance historique est directement proportionnelle à celle de (sa) culture personnelle $»^{43}$, et accepter que l'histoire soit celle, particulière, de l'historien. Car:

\begin{abstract}
L'image que chaque historien donne du passé est si profondément, si organiquement modelée par sa personnalité que leurs différents points de vue sont en définitive moins complémentaires qu'exclusifs.

A l'idéal illusoire de la «connaissance valable pour tous» j'opposerai celui de la vérité valable pour moi, et j'y verrai une garantie de sérieux, d'exigence, de rigueur ${ }^{44}$.

La critique s'indigne et proteste contre cette invasion du moi haïssable. L'honnêteté scientifique me parait exiger que l'historien par un effort de prise de conscience, définisse l'orientation de sa pensée, explicite ses postulats (dans la mesure où la chose est possible); qu'il se montre en action et nous fasse assister à la genèse de son œuvre: pourquoi et comment il a choisi et délimité son sujet; ce qu'il cherchait, ce qu'il a trouvé; qu'il décrive son itinéraire intérieur, car toute recherche historique, si elle est vraiment féconde, implique un progrès dans l'âme même de son auteur... en un mot qu'il fournisse tous les matériaux qu'une introspection scrupuleuse peut apporter à ce qu'en termes empruntés à Sartre j'avais proposé d'appeler sa «psychanalyse existentielle ${ }^{45}$.
\end{abstract}

\title{
Le dilemme du psychologue américain C.R. Rogers
}

Le cas de ce psychologue américain est intéressant parce qu'il explicite le conflit entre l'attitude de distanciation du scientifique et les besoins d'empathie du psychothérapeute. Rogers a le plus grand respect pour la science, vue à travers le positivisme américain. Devenu psychothérapeute, il découvre un mode d'interaction et de communication avec ses patients tout à fait différent de celui qu'il connaissait en tant que scientifique classique. Plus il accepte de s'oublier, avec les risques éventuels de l'aventure, plus il accepte le patient en tant que personne, et non en tant qu'objet d'études, et meilleur il est thérapeute. Il découvre ainsi le pouvoir de vérité d'une posture existentielle (il cite Kierkegaard et Buber), s'opposant à la posture du scientifique, dont l'esprit d'objectivité se révèle être un obstacle à la conduite de la thérapie. Il tente de mieux cerner ce conflit personnel, intérieur, dans un texte qui, d'abord écrit pour lui-même, sera ensuite publié ${ }^{46}$.

Dans une première section, il dégage l'essence de la thérapie, où il insiste sur l'importance de l'expérience vécue intérieurement, subjectivement, par les deux protagonistes, thérapeute et client. Dans la seconde, il regarde la thérapie avec les yeux du scientifique, qui tente d'opérationnaliser, pour pouvoir les mesurer, les pratiques thérapeutiques, par exemple, à travers la proposition suivante: «L'acceptation du client par le thérapeute conduit à l'acceptation de sa personnalité par le client ». Il imagine comment on pourrait mesurer - à travers des techniques psychologiques et/ou des examens physiologiques - ces acceptations et leur variation avant et après la cure.

\footnotetext{
Op. cit., p. 34.

Op. cit., p. 213.

Op. cit., p. 231.

46 C. R. Rogers, Persons or Science? A Philosophical Question in On Becoming a Person. A Therapist's View of Psychotherapy, Boston, Houghton Mifflin Company, 1961.
} 
Le dialogue entre l'«expérientialiste» et le scientifique se poursuit à travers des questions; le scientifique pose la question de la validité de la connaissance subjective, remarque l'absence de toute méthode partagée, le manque de toute prédictibilité, et s'inquiète du bien-fondé du rejet de la démarche scientifique qui a fait ses preuves dans tant de domaines. L' «expérientialiste» pense que cette démarche est, au mieux, sans pertinence pour le type de connaissance et de pratique qui l'intéresse, au pire, l'en empêche, et risque par ailleurs de conduire à la manipulation des personnes. Il pose que l'éthique, qui intervient de façon particulièrement profonde en psychothérapie, est plus importante que la science.

Une année plus tard, Rogers trouve la solution de son dilemme, après de nombreuses discussions avec des étudiants, des collègues, des amis. Cette solution, c'est, de fait, le dépassement du scientisme où il se trouvait de par sa formation, et le remplacement de l'idée étriquée qu'il se faisait de la science par quelque chose de plus vaste.

Il découvre que la science n'est pas seulement quelque chose d'extérieur, qu'on écrit avec un grand $\mathrm{S}$, une collection de savoirs objectifs et de méthodes neutres organisées méthodiquement, vue qui ne peut qu'entraîner la dépersonnalisation et les risques de manipulation. La science réelle s'ancre dans les individus qui la font. La subjectivité est donc essentielle à la construction de la science. Cette subjectivité apparaît dans toutes les phases de la science classique. A la phase créative, le scientifique s'immerge dans son objet d'étude d'une manière comparable à ce que fait le thérapeute, sentant plus qu'il est en mesure d'expliciter. Puis il doit vérifier que son intuition ne l'a pas trompé, en choisissant les méthodes de contrôle adéquates. Ses résultats sont intéressants dans la mesure où ils peuvent le porter plus loin, personnellement, dans la démarche de connaissance. S'ils ne sont là que pour être reconnus par les autres, c'est le signe d'une insécurité psychique personnelle ${ }^{47}$. La subjectivité intervient également lorsqu'il discute et confronte sa perception de la réalité avec les autres, gage d'une vérification intersubjective. C'est avec ceux qui sont prêts à accepter les règles de base de l'investigation qu'il va chercher à communiquer, pas avec les autres. Enfin, il y va de sa responsabilité que les résultats de la connaissance qu'il obtient soient utilisés pour le bien ou le mal de l'humanité. Ce n'est pas la science qui utilise à mauvais escient ses résultats, ce sont des personnes.

C'est en relevant les aspects de subjectivité présents dans la science classique que Rogers obtient la résolution de son conflit personnel. Il retrouve l'unité de sa personne, en admettant qu'elle puisse fonctionner selon divers modes : thérapeute, il s'implique dans une expérience unique, vécue plutôt qu'examinée, dans un mode de conscience non réflexif, où il est participant plutôt qu'observateur. Mais parce qu'il est curieux de l'ordre délicat qui semble exister dans l'univers et dans cette relation, il peut s'abstraire de cette expérience et la regarder en tant qu'observateur, se faisant lui-même et les autres objets de son observation.

La résolution du conflit a donc été obtenue en mettant «la personne subjective, existentielle, avec les diverses valeurs qui l'animent, au fondement et à la racine de la relation thérapeutique et de la relation scientifique».

\footnotetext{
47 On pense ici au travail de Devereux pour qui l'insistance sur les méthodes en sciences sociales permet d'évacuer l'angoisse d'avoir à se confronter directement aux gens. G. Devereux, De l'angoisse à la méthode dans les sciences du comportement, Paris, Flammarion, 1980.
} 


\section{Les ethnologues}

Les ethnologues sont ceux qui ont reconnu le plus facilement l'implication de leur propre personne dans leur travail professionnel, au point que, pour certains, il est à peu près impossible de distinguer l'un de l'autre. C'est ainsi que G. Balandier écrit, à propos de l'anthropologue $\mathrm{J}$. Goody ${ }^{48}$ :

Il (l'anthropologue) doit parler à son tour, se mettre en jeu dans une façon de confession où l'expérience personnelle se sépare mal de ce qui vient de la pratique scientifique. Il révèle ainsi combien la richesse de l'une conditionne la fécondité de l'autre.

et encore:

Expliquer des peuples étrangers chez qui l'on a vécu, et que l'on a aimés, c'est inévitablement s'expliquer soi-même. Il y a dans l'analyse de telles relations, même lorsqu'elles gardent un caractère scientifique, la révélation d'une aventure personnelle. Je crois possible, en ouvrant l'œuvre des ethnologues, de repérer les principales étapes de leur propre histoire. Dès qu'ils élargissent la recherche, ils enrichissent en même temps cette autobiographie qui se développe en contrepoint de leurs travaux. Un tel résultat ne demeure pas toujours caché, mais il n'apparaît en pleine lumière qu'exceptionnellement: ainsi, dans cette Afrique fantôme, par laquelle Michel Leiris annonça sa carrière d'africaniste, ou encore dans Tristes tropiques, où Claude Lévi-Strauss, retrouvant la tradition du voyage philosophique, se situe par rapport à sa profession.

... l'accès à une civilisation étrangère et cette confiance, qui se gagne par une patiente compréhension, sont plus le résultat d'une ascèse que d'un jeu supérieur ou d'une technique scientifique ${ }^{49}$.

\section{Être affecté}

L'ethnologue J. Favret-Saada est allée étudier les pratiques de sorcellerie dans le bocage. Au cours de cette étude, elle s'est trouvée «prise» dans ces pratiques, au point d'aller voir, pour se libérer, une désensorcelleuse. Cette expérience, toute contraire aux pratiques de distanciation de la science, l'a conduite à une réflexion épistémologique, puisque c'est en acceptant de perdre le contrôle d'elle-même qu'elle a abouti à un savoir qu'elle n'aurait pas obtenu sinon ${ }^{50}$.

D'abord, quelques réflexions sur la façon dont j'ai obtenu mes informations sur le terrain: je n'ai pu faire autrement que d'accepter de m'y laisser affecter par la sorcellerie, et j'ai mis en place un dispositif méthodologique tel qu'il me permette d'en élaborer après coup un certain savoir.

En effet, ce qu'on lui laissait entendre, c'est ceci:

La sorcellerie, ceux qui n'y sont pas pris, ils ne peuvent pas en parler.

Ils ne m'en ont donc parlé que quand ils ont pensé que j'y étais «prise», c'est-à-dire quand des réactions échappant à mon contrôle leur ont montré que j'étais affectée par les effets réels - souvent dévastateurs - de telles paroles et de tels actes rituels... En fait, ils exigeaient de moi que j'expérimente pour mon compte personnel - pas celui de la science - les effets réels de ce réseau particulier de communication humaine en quoi consiste la sorcellerie.

\footnotetext{
48 G. Balandier, «Le Monde», 7 juin 1996.

49 G. Balandier, Afrique ambiguë, Paris, Plon, 1983 (1957), p. 6 et p. 8.

50 J. Favret-Saada, «Gradhiva», 1990, n 8, pp. 3-9.
} 
Cette expérience particulière exige que l'on prenne des risques pour soimême, mais aussi pour son projet de connaissance, tant les deux aventures sont distinctes.

Accepter d'être affecté suppose toutefois qu'on prenne le risque de voir s'évanouir son projet de connaissance. Car si le projet de connaissance est omniprésent, il ne se passe rien. Mais s'il se passe quelque chose et que le projet de connaissance n'a pas sombré dans l'aventure, alors une ethnographie est possible.

Favret-Saada choisit «de donner un statut épistémologique à ces situations de communication involontaire et non intentionnelle» qui n'appartiennent, pour elle, ni aux catégories classiques «de l'observation participante, ni surtout de l'empathie $»$.

Elle souligne un «trait distinctif de cette ethnographie: elle suppose que le chercheur tolère de vivre dans une sorte de schize. Selon les moments, il fait droit à ce qui, en lui, est affecté, malléable, modifié par l'expérience du terrain; ou bien à ce qui, en lui, veut enregistrer cette expérience, veut la comprendre, en faire un objet de science». On notera que, arrivé à cet instant de sa réflexion, Rogers acceptait comme une unification de sa personne de pouvoir vivre, selon les moments, ses deux personnalités, et il n'aurait sans doute pas utilisé le terme de schize qui a une connotation douloureuse.

\title{
Un exercice spirituel
}

Balandier parlait d'ascèse, Bourdieu rejoint Marrou quand il parle, au sujet de l'entretien compréhensif qu'il mène, d'exercice spirituel ${ }^{51}$ :

\begin{abstract}
Au risque de choquer aussi bien les méthodologues rigoristes que les herméneutes inspirés, je dirais volontiers que l'entretien peut être considéré comme une forme d'exercice spirituel, visant à obtenir, par l'oubli de soi, une véritable conversion du regard que nous portons sur les autres dans les circonstances ordinaires de la vie. La disposition accueillante, qui incline à faire siens les problèmes de l'enquêté, l'aptitude à le prendre et à le comprendre tel qu'il est, dans sa nécessité singulière, est une sorte d'amour intellectuel: un regard qui consent à la nécessité, à la manière de l'«amour intellectuel de Dieu », c'est-à-dire de l'ordre naturel, que Spinoza tenait pour la forme suprême de la connaissance.
\end{abstract}

\section{Les interventions psycho-sociologiques}

Je terminerai cette section en évoquant rapidement les diverses positions que choisissent, de façon, là encore, plus ou moins explicite, les intervenants psychosociologiques.

Je m'appuie sur l'ouvrage de J. Dubost ${ }^{52}$. Dubost a réfléchi s'il lui fallait utiliser le terme de science pour ses pratiques d'intervention dans la société. S'il décide finalement de le faire, tout conscient qu'il soit de l'inadéquation du terme par rapport aux exigences fortes qu'il implique, c'est pour que «le travail produit par le processus prenne sens en un point qui soit en quelque sorte extérieur aux partenaires de la relation ».

\footnotetext{
51 P. Bourdieu et al., La misère du monde, Paris, Seuil, 1993, pp. 913-914.

52 J. Dubost L'intervention psychosociologique, Paris, PUF, 1987, p. 146 et p. 326.
} 
Dubost classe les différents types d'intervention selon les buts que se donnent les intervenants: il peut s'agir de fonctions adaptatrice, critique, ou d'élucidation pure. Dans le même esprit, J.Maisonneuve (1972) avait parlé de tendances orthopédique, démiurgique, maïeutique.

Il faudrait ici encore évoquer les divers termes utilisés par les psychanalystes: échange entre deux inconscients, écoute flottante...

C'est tout un monde intérieur, d'une grande richesse, et dont seuls certains aspects se laissent décrire et peuvent éventuellement fournir des pistes de méthode.

\title{
III. L'IDENTITE ÉPISTÉMIQUE
}

\author{
Il est rare que des géomètres soient fins et \\ que les fins soient géomètres... Mais les esprits \\ faux ne sont jamais ni fins ni géomètres... \\ Pascal. Différence entre l'esprit de géomé- \\ trie et l'esprit de finesse
}

Pascal, pour avoir ainsi défini deux sortes d'esprit, l'esprit de géométrie et l'esprit de finesse, pourrait être reconnu comme le précurseur d'une discipline qui étudierait ce que je nomme l'identité épistémique et qui, tenant à la fois de l'épistémologie et de la psychologie, pourrait s'intituler «psycho-épistémologie».

D'autres termes, plus ou moins voisins, existent: certains parlent d' «idiosyncrasie», qui est cependant plus général. Merton indique, dans le volume qui a été dédié à Lazarsfeld, que ce dernier «a souffert toute sa vie d'identité cognitive ${ }^{53}$ (mais, à mon avis, pas d'identité épistémique). L'on parle aussi couramment de «style», et comme on le sait, «le style, c'est l'homme». Un psychologue (voir plus loin) parle des «intelligences multiples». Je vois personnellement le terme que j'ai choisi comme une propriété profondément ancrée dans la personne, structurelle en quelque sorte, et qui ne change pas aisément.

Ce texte voudrait engager chacun à tenter de mieux connaître ses propres façons de penser. C'est pourquoi, plutôt que d'utiliser les termes styles, ou cadres de pensée, ou types, ou tout autre terme descriptif, qui impliquerait un observateur objectif, j'utilise le terme «identité » qui a l'avantage, à mes yeux, d'attirer l'attention sur la prise de conscience de soi-même.

\section{L'identité}

Le terme «identité » est difficile à définir, et pourtant très présent ${ }^{54}$. Le psychologue américain E. H. Erikson est le premier à avoir introduit une réflexion approfondie sur la notion; il considère, à cette époque (1950) que «l'étude de l'identité devenait aussi centrale que celle de la sexualité à l'époque de Freud.» Et

\footnotetext{
53 J. Lautman et B.-P. Lécuyer, dir. Paul Lazarsfeld (1901-1976), La sociologie de Vienne à NewYork, Paris, L'Harmattan, 1998.

54 P. Tap s'est efforcé de donner une définition précise de la notion, en la décomposant en sept «dimensions ». P.Tap, dir. Identité individuelle et personnalisation. Privat, 1980.
} 
pourtant, dans la préface de son livre Adolescence et crise; la quête de l'identité, il dit: «Plus on écrit sur ce thème et plus les mots s'érigent en limite autour d'une réalité aussi insondable que partout envahissante $»^{55}$.

Certains refusent la notion (Hume, Fumaroli...) en insistant sur le côté labile des phénomènes de conscience. D'autres s'en serviront (James...). Nul doute qu'il doit en être de même pour la notion d'identité épistémique. Si je la trouve moi-même utile, c'est que j'ai pu en faire l'expérience pour mon propre compte. Je réfère le lecteur au film de Woody Allen, Zelig, entièrement construit autour d'un personnage souffrant de ce manque d'identité. De fait, pour ceux qui n'ont pas d'identité et en souffrent médiocrement, ils ne peuvent savoir ce que c'est. Pour ceux qui en ont une et en repèrent les fluidités, ils peuvent préférer insister sur ces fluidités. C'est pourquoi, me semble-t-il, que c'est lors du passage d'une identité flottante à une identité mieux assurée que se fait le mieux la perception de cette notion. Arrivé/e à un palier où l'angoisse de l'incertitude est moindre, l'on peut se risquer de nouveau à s'essayer dans une ou plusieurs directions.

\section{Les «formes de l'intelligence» de Gardner}

L'ouvrage d'H. Gardner, a connu un certain succès en France. Il s'agit de la traduction d'un livre qui date de 1983, et dont le titre anglais approche mieux l'idée qui est débattue ici: «Frames of mind», ce sont les «cadres de l'esprit ${ }^{56}$. Le titre français a pour mérite - et c'était peut-être là le but - de tordre le cou à une certaine idée de l'intelligence avant tout scolaire, comme on aime à la pratiquer et la développer en France. C'est ainsi qu'en dehors des intelligences verbales (correspondant aux disciplines littéraires) et logico-mathématiques (correspondant aux «sciences»), l'auteur introduit, et donc légitime, des intelligences musicales, corporelles, et même l'intelligence de soi et des autres. L'auteur, interviewé dans l'Express, rajoute même «l'intelligence existentielle».

Si l'on prend, comme semble le faire l'auteur, ces cadres de l'esprit comme des données psycho-biologiques, alors, c'est à partir de ces intelligences multiples que se bâtira évidemment l'identité épistémique, qui porte plus spécifiquement sur les manières de connaître et de comprendre.

\section{Les types épistémologiques de Maruyama}

Un chercheur japonais, M. Maruyama, rencontré dans des congrès, m'a donné deux articles de lui concernant ce qu'il nomme «les types épistémologiques » ou encore «mindscapes», autrement dit, les paysages mentaux ${ }^{57}$. Ces articles comportent de nombreuses références, provenant de champs très divers: psychologie cognitive, sociologie de la culture, sans oublier les auteurs bien connus de l'épistémologie contemporaine, tels Kuhn ou Geertz.

\footnotetext{
55 E. H. Erikson, Adolescence et crise ; la quête de l'identité, Paris, Flammarion, 1972, p. 5.

56 H. Gardner, Les formes de l'intelligence, Paris, Ed. Odile Jacob, 1997.

57 M. Maruyama, Mindscapes and Science Theories, in «Current Anthropology », 21, 1980, pp. 589599; Epistemological heterogeneity and subsedure : individual and social processes, in «Communication and Cognition», 24, 1991, pp. 255-272.
} 
Pour lui, ces «types épistémologiques » seraient formés pendant l'enfance, et il serait très difficile de les changer ultérieurement. D'où les difficultés de compréhension entre personnes relevant de «types» différents.

Son travail, brassant de nombreux domaines spécialisés, consiste à établir une classification de base, dont les éléments peuvent être croisés afin d'introduire plus de complexité, et d'examiner, à partir de là, quels types de positions ces caractéristiques induisent dans des domaines de connaissance ou d'action divers, allant de la philosophie aux théories scientifiques, en passant par la prise de décision, l'«architecture, les valeurs, les positions concernant la logique ou la causalité ».

Voilà donc, au départ, une idée très voisine de la mienne et qui, pourtant, se développe dans des directions et dans un style qui éveillent un certain malaise en moi. Je n'arrive pas à «croire» véritablement ni à cette classification, trop poussée, ni aux modèles épistémologiques qu'elle utilise, trop lâches par rapport à la réalité de la science que je connais. Autrement dit, ces modèles, qui prennent si bien l'apparence de la science, me semblent être poussés trop loin pour pouvoir être utiles. C'est comme s'il valait mieux, parfois, accepter d'utiliser des notions sans qu'elles soient trop bien définies, leur manque de précision leur donnant une sorte de volant de sécurité dans l'approche juste (pertinente) de domaines nécessairement flous.

\title{
Eviter les discussions sans issue
}

La reconnaissance de ces identités différentes pourrait permettre d'éviter quelques malentendus dans les discussions, et donc, peut-être, d'aller plus loin dans celles-ci. Déjà Socrate, s'inquiétant de savoir comment peut se faire la communication entre personnes, signalait qu'une technique de l'art de parler ne pourrait se développer qu'après «avoir fait une classification des espèces de discours aussi bien que des espèces d'âmes et de leurs caractéristiques respectives ${ }^{58}$. Quand Wittgenstein remarque, en passant: «Hegel me semble toujours vouloir dire que des choses qui ont l'air différentes sont en réalité les mêmes. Alors que ce qui m'intéresse est de montrer que des choses qui ont l'air les mêmes sont en réalité différentes ${ }^{59}$, je croirais qu'il y a bien là un moyen de contourner certaines discussions stériles.

\section{Chez les mathématiciens}

H. Poincaré est de ceux qui trouvent important d'accepter que des discussions ne puissent avoir lieu, parce que les «âmes» sont dissemblables.

\begin{abstract}
De tous temps, il y a eu en philosophie des tendances opposées et il ne semble pas que ces tendances soient sur le point de se concilier. C'est sans doute parce qu'il y a des âmes différentes et qu'à ces âmes nous ne pouvons rien changer. Il n'y a donc aucun espoir de voir l'accord s'établir entre les pragmatistes et les cantoriens. Les hommes ne s'entendent pas parce qu'ils ne parlent pas la même langue et qu'il y a des langues qui ne s'apprennent pas ${ }^{60}$.
\end{abstract}

58 Platon, Phèdre, Euvres complètes, T.2, Paris, Gallimard, 1950, p. 69.

59 Cité par J. Bouveresse: Philosophie, mythologie et pseudo-science. Wittgenstein lecteur de Freud, 1991, Editions de l'Eclat, p. 10.

60 H. Poincaré, Dernières Pensées, Paris, Flammarion, 1963 (1913), p. 95, cité par A.-F. Schmid, Une philosophie de savant: Henri Poincaré et la logique mathématique, François Maspero, 1975, pp. 155-156. 
Il fait ici allusion à sa querelle avec Russell, dont il n'accepte point les travaux de logique trop poussés pour définir les mathématiques, lui-même défendant la thèse de l'intervention de l'intuition dans cette discipline.

M. Russell me dira sans doute qu'il ne s'agit pas de psychologie, mais de logique et
d'épistémologie; et moi, je serai conduit à répondre qu'il n'y a pas de logique et d'épis-
témologie indépendantes de la psychologie; et cette profession de foi clora probablement
la discussion parce qu'elle mettra en évidence une irrémédiable divergence de vues ${ }^{61}$.

Le mathématicien J. Hadamard s'est exercé à explorer la psychologie de l'invention dans le domaine mathématique ${ }^{62}$. Il remarque, lui aussi, qu'il existe, sur plusieurs problèmes de la pensée «deux catégories d'esprit dont les uns nient ce qui semble évident aux autres et dont chaque catégorie croit constater la déraison chez l'autre et la prend en pitié ». Un des problèmes qu'il considère concerne les rapports entre pensée et langage intérieur, l'autre porte sur la possibilité de fonder la morale sur la science. Il est là-dessus de l'avis de Poincaré sur son impossibilité, tandis que «le grand philosophe Emile Durkheim» est de l'avis contraire, et accueille son opinion par une phrase du genre suivant: «Vous allez voir qu'il va encore dire des bêtises » (sic).

L'acceptation de l'existence de désaccords bien tranchés contraste avec l'étonnement désapprobateur d'un R. Aron présentant les thèses de Max Weber sur la «guerre des dieux ${ }^{63}$. Elle rappelle aussi l'âpre bataille qui a eu lieu autour de l'affaire Sokal ${ }^{64}$. Comme le signale Jurdant dans l'introduction du livre répliquant à celui de Sokal et Bricmont, la remise en cause d'équilibres instables (à savoir l'ignorance réciproque entre scientifiques d'une part, philosophes et spécialistes des sciences de l'homme de l'autre) a provoqué des blessures qui concernent plus les «identités » elles-mêmes que les idées ${ }^{65}$.

Il n'est sans doute pas étonnant de trouver chez des mathématiciens comme Hadamard, Poincaré, (peut-être aussi chez le juriste de formation qu'a été Weber) des visions tranchées sur l'existence de positions incompatibles, et qu'il est plus simple de reconnaître telles. C'est qu'ils ont l'habitude d'avoir affaire à des situations dichotomiques: on a démontré ou pas un théorème, cette conjoncture est vraie ou fausse... On peut par ailleurs penser que le choix des mathématiques comme discipline n'est pas étranger à cette forme d'esprit, auquel d'autres vont rechigner. En outre, puisque les sciences exactes se construisent autour d'objets reconnus par tous, il est plus facile que dans d'autres disciplines de détecter les identités intellectuelles de ceux qui y participent.

C'est ainsi qu'en mathématiques, il est coutume de distinguer les esprits intuitifs et les esprits rigoureux, étant donné qu'ils se complètent bien dans le travail, puisqu'on a besoin à la fois d'imagination et de logique. L. Schwartz, Médaille Fields (l'équivalent du prix Nobel pour les mathématiques) pour sa découverte

\footnotetext{
61 H. Poincaré, op.cit. p. 31, cité par A-F. Schmid, p. 89.

62 J. Hadamard, Essai sur la psychologie de l'invention dans le domaine mathématique, GauthierVillars, 1975, pp. 132-133.

63 R. Aron, Préface à M. Weber, Le savant et le politique, Paris, UGE, 1986.

64 J. Feldman, L'affaire Sokal, op. cit.

65 B. Jurdant, dir., Impostures scientifiques..., op. cit..
} 
des «distributions », sait décrire avec précision ses qualités et défauts intellectuels ${ }^{66}$. Il parle ainsi de son «esprit lent», qui va avec « le besoin absolu de... comprendre à fond»; de son «excellente mémoire», de «la confiance en soi (qui) conditionne le succès »; de la «vacuité de son hémisphère droit» qui le rend «incapable de se repérer dans l'espace» - raison pour laquelle il n'a jamais pu apprendre à conduire, et qui le fait s'étonner de l'amour qu'il porte pourtant à la géométrie, approchée, il est vrai, du côté analytique plutôt que visuel; de son «esprit mathématique, profondément classificateur», ce qui lui donne sa place comme membre de Bourbaki, ce collectif de mathématiciens qui s'est chargé de reprendre à la base les mathématiques et de les asseoir solidement sur la méthode axiomatique; de son exigence de la simplicité, qui le fait reprendre une démonstration jusqu'à en trouver le chemin le plus direct. J'ajouterai à ce tableau la force de la relation à la vérité: "Il est naturellement impossible de ne jamais mentir. Mais, quand je suis obligé de ne pas dire la vérité, je me la dis toujours, sans exception, à moi-même. Autrement dit, je sais exactement ce que je pense et, si je suis obligé de déformer ma parole, je m'efforce du moins que ma pensée reste intègre ». Nous sommes bien ici dans l'esprit de géométrie.

\section{Les thêmata de Holton}

Cette notion, proposée au départ par le philosophe des sciences I. Lakatos, a fait l'objet de recherches poussées par Holton ${ }^{67}$. Le travail de Holton, d'une grande finesse et d'une grande érudition, montre que certains thèmes de la physique sont constants, et mobilisent l'énergie, l' «attention, voire, la passion, de certains physiciens». Examinant la pensée de certains physiciens - parmi les «grands »-il y découvre certains invariants, la défense de certains principes, qui contribuent à expliquer leur œuvre. Il rajoute ainsi aux diverses composantes explicatives de la physique - il en distingue huit autres, concernant l'histoire, la sociologie, l'épistémologie... - ces caractéristiques plus personnelles, et qui relèvent de ce que je nomme la psycho-épistémologie. Holton met en garde, par ailleurs, contre la tentation du réductionnisme qui ramènerait la physique à cette seule composante-là, oubliant son autonomie épistémologique, qu'il défend contre les post-modernes.

Il s'agit bien d'éléments ancrés profondément dans la personnalité psychoépistémique. Holton parle de «position tranchée, d'engagement précoce, inébranlable», et également de structures et régularités sous-jacentes. Ces convictions profondes sont acquises comme une «empreinte» (le terme est de moi, ici) qui s'incruste dans un cerveau encore vierge, pour y durer, de la même façon «qu'on aime pour la première fois ».

Entendons, par exemple, M. Von Laue et M. Born parler d'un physicien, Abraham, dont ils ont rédigé la nécrologie ${ }^{68}$.

\footnotetext{
L. Schwartz, Un mathématicien aux prises avec le siècle, Paris, Ed. Odile Jacob, 1997.

67 G. Holton, Aspects thématiques de la pensée scientifique, in «Le Débat», 1980; n 4, pp. 89-115; L'imagination scientifique, Paris, Gallimard, 1981.

68 Op. cit., pp. 97-98.
} 
Il témoignait une révulsion viscérale pour les abstractions d'Einstein; il aimait son éther absolu... ainsi qu'on aime pour la première fois quand on est jeune, un amour qu'aucune expérience ne pourra effacer par la suite... Son opposition se fondait sur des convictions physiques, fondamentales, auxquelles il resta attaché aussi longtemps qu'il put, simplement pour leur conformité à sa sensibilité... Ainsi qu'il l'avait lui-même indiqué, à l'occasion, il n'avait aucun argument à opposer aux cohérences logiques; il les admettait, et les admirait, comme étant la seule issue possible au plan de la relativité généralisée. Mais c'était un plan auquel il répugnait profondément, et il espérait que l'observation astronomique viendrait l'infirmer, et remettre en honneur le vieil éther absolu.

Ainsi, écrit Holton, «une des fonctions essentielles d'un thêma est de servir à rendre le monde intelligible d'une manière que les impératifs de la logique seule ne sauraient permettre», puisqu'elles «ne sont, en tant que telles, ni vérifiables ni réfutables». Elles sont, pour lui, «une des sources d'énergie primordiales de l'élan novateur».

Ces conceptions premières procèdent le plus souvent par couples, et sont relativement peu nombreuses. Il y a ainsi l'atomisme et le continu; l'analyse et la synthèse; le réductionnisme et le holisme; l'invariance et l'évolution... Holton remarque à son tour la difficulté, voire, l'impossibilité de s'entendre à leur sujet.

\begin{abstract}
Entre de tels contraires thématiques, il n'y a pas de façon simple d'établir un consensus. Werner Heisenberg fut de ceux qui s'évertuèrent à convaincre Einstein... «C'est une excellente après-midi que je passai avec Einstein, mais quand nous en vînmes à l'interprétation de la mécanique quantique, je demeurai incapable de le convaincre, et lui incapable de me convaincre... il disait toujours: 'Soit, je suis d'accord que toute expérience, dont les résultats sont calculables au moyen de la mécanique quantique, se déroulera comme vous l'indiquez, mais il reste qu'un tel schème ne peut être une description ultime de la Nature'» ${ }^{69}$.
\end{abstract}

La querelle ici, consiste en l'apparition, à la base des formules de la mécanique quantique, de la notion de probabilité. Un des thêmata - pas vraiment souligné, me semble-t-il, par Holton - est le couple certitude/probabilité. Certains scientifiques, comme on sait, se sont violemment opposés au Calcul des probabilités, la science consistant, pour eux, à aboutir à des certitudes: d'Alembert, par exemple, Comte, Claude Bernard, pour ne citer que quelques-uns. Pour en revenir à la mécanique quantique, Einstein refusait de penser que Dieu puisse jouer aux dés.

\title{
Du côté des sciences de l'homme
}

Si l'on passe à présent au cas des sciences humaines, on se trouve dans une situation différente, la complexité des approches, l'absence de possibilité de décider entre le vrai et le faux, ne favorisant pas ces positions tranchées. Et, comme je l'ai déjà dit, on peut penser que ceux qui les aiment vont plutôt choisir les sciences exactes. Les positions fondamentales, difficiles à expliciter, feront souvent l'objet, plutôt que de désaccords francs, de coexistences polies.

Freud est l'un des penseurs qui a sans doute été le plus étudié, en raison de l'originalité de sa pensée et du succès considérable qu'elle a eu. P.-L. Assoun a étudié cette pensée ${ }^{70}$, et utilise le terme «identité épistémique freudienne», c'est-

\footnotetext{
69 Op. cit., p. 108.

70 P.-L. Assoun, Introduction à l'épistémologie freudienne, Paris, Payot, 1981.
} 
à-dire, explique-t-il, «l'épistémologie rigoureusement indigène et immanente de connaissance qui appartient à Freud ». Il examine avec soin comment cette pensée s'est développée, en contact avec les idées de son temps et, à travers les influences reçues, comment elle a su tracer son propre chemin. Le fait qu'il s'agisse d'un développement me paraît distinguer le sens de cette expression de l'emploi que personnellement j'en fait.

Ce que je caractériserais plutôt comme identité épistémique de Freud, je le trouverais par exemple sur les rapports que Freud lui-même dit avoir avec la philosophie: tout comme Mach, qui l'a profondément influencé dans la vision qu'il a eu de la science, il admet ne pas être à l'aise dans les généralisations spéculatives de type philosophique.

On pourrait aussi remarquer la clarté de sa pensée, la volonté de faire science, qui le conduit parfois au scientisme, une certaine attitude dogmatique, dans le sens où sont mis en place des modèles, des explications, des théories, bref, ce qui constitue un discours de maîtrise. On peut retrouver là un habitus qui proviendrait peut-être de sa formation première de biologiste.

Assoun note aussi le holisme de Freud, c'est-à-dire la position qui consiste à affirmer qu'il n'y aurait qu'une seule science, qu'il s'agisse des sciences exactes ou de la science du psychisme qu'il développe. Il peut peut-être s'agir, en effet, d'un trait épistémique profond, personnel, qui plonge loin ses racines. Ou bien d'une conviction plus superficielle, qui serait une façon de légitimer son travail comme scientifique, dans le scientisme ambiant de la fin du $19^{\text {ème }}$ siècle. Je ne suis pas en mesure de me prononcer là-dessus.

R. Aron, en présentant l'œuvre des grands penseurs de la sociologie, nous fait part de ce qui relève de ses sentiments personnels par rapport à la pensée de certains d'entre eux ${ }^{71}$ :

Je crains d'avoir été injuste à l'égard d'Emile Durkheim pour la pensée duquel j'ai tou-
jours ressenti une immédiate antipathie. Max Weber ne m'irrite jamais même quand je lui
donne tort, alors qu'il m'arrive d'éprouver un sentiment de malaise même quand les
arguments de Durkheim me convainquent. Je laisse aux psychanalystes et aux socio-
logues le soin d'interpréter ces réactions probablement indignes d'un homme de science.

Ainsi, ces sentiments sont si profonds qu'Aron ne cherche point à en trouver les raisons, il en délègue la tâche au psychanalyste (et au sociologue). Dans sa Leçon inaugurale au Collège de France, il revient là-dessus, évoquant son «allergie à la pensée durkheimienne» et son «affinité élective avec la pensée de Max Weber $»^{72}$.

R.Boudon ${ }^{73}$ utilise également les termes de «sympathie» ou d' «antipathie» quand il parle de ses choix intellectuels.

Je me suis toujours reconnu dans une conception scientifique de la sociologie. Cette préférence peut être rattachée à certaines expériences de sympathie ou d'antipathie intellectuelle datant du temps de mes études supérieures. Ces sentiments étaient eux-mêmes liés

\footnotetext{
R. Aron, Les étapes de la pensée sociologique, Paris, Gallimard, 1967, pp. 20-21.

R. Aron, De la condition historique du sociologue, Paris, Gallimard, 1971.

73 R. Boudon, Pourquoi devenir sociologue? Réflexions et évocations, in «Revue Européenne des sciences sociales », $\mathrm{n}^{\circ} 120,2001$, p. 27.
} 
à l'impression de clarté ou au contraire d'opacité que j'ai éprouvée à la lecture de tel ou tel auteur... J'ai ressenti pour d'autres auteurs une antipathie immédiate, notamment pour ceux qui voient le sujet social comme muni d'une conscience fausse par essence... Mais cette antipathie ne s'est transformée en rejet que lorsqu'elle était à la fois intellectuelle et morale.

Il défend la clarté, et rejette la séduction, la manipulation, la verbosité... F. Furet a étudié longuement la pensée de Tocqueville dont il souligne l'originalité ${ }^{74}$.

Il offre l'exemple limite d'un intellectuel qui n'a jamais «appris » que dans le cadre de ce qu'il avait préalablement pensé, ce qui lui donne à la fois... une extraordinaire étroitesse et une exceptionnelle profondeur.

Tocqueville écrivait en effet à un ami, en 1835: «Il y a dix ans que je pense une partie des choses que je t'exposerai tout à l'heure. Je n'ai été en Amérique que pour m'éclairer sur ce point ${ }^{75}$. Son voyage en Amérique lui a ainsi servi d'une sorte de mise à l'épreuve de ses idées.

Tocqueville, écrit encore Furet, «est un esprit qui laboure indéfiniment les mêmes idées et qui en déterre toujours des aspects nouveaux ${ }^{76}$. Ces idées sont très simples, au départ, il s'agit d'un système à deux dimensions, note Furet: le principe de la noblesse est vaincu par le principe de la démocratie. Il pose «comme un axiome» que la démocratie ne peut que se développer partout, et il va en examiner le fonctionnement là où elle est «chimiquement pure », en Amérique.

Or, Tocqueville vient lui-même d'une famille aristocratique, profondément blessée par les événements en cours. C'est ce «socle existentiel», selon la formule de Furet, qui est la base de la perception du système et de la volonté d'en déceler toutes les conséquences, comme une sorte de fascination malheureuse dont on ne peut sortir que par un travail de raison. Tocqueville présente ainsi son livre:

Le livre entier qu'on va lire a été écrit sous l'impression d'une sorte de terreur religieuse produite dans l'âme de l'auteur par la vue de cette révolution irrésistible qui marche depuis tant de siècles à travers tous les obstacles, et qu'on voit encore aujourd'hui s'avancer au milieu des ruines qu'elle a faites ${ }^{77}$.

Furet insiste sur cette façon de «conceptualiser son expérience», remarquant que cela «le sépare de la plupart des grands esprits philosophiques, formés surtout par l'étude abstraite des doctrines et des idées », mais que cela explique aussi «son obstination à creuser une seule idée, qu'on peut bien appeler, comme on dit d'une femme, celle de sa vie $\gg^{78}$.

Le besoin de partir ainsi de principes simples, voire d'un principe unique, se reflète dans cette lettre de Tocqueville à un ami: «En Amérique, toutes les lois

\footnotetext{
74 F. Furet, Le système conceptuel de la «démocratie en Amérique», Préface à A. de Tocqueville, De la démocratie en Amérique, I, Paris, Garnier-Flammarion, 1981.

75 A. de Tocqueville, Lettre à Kergolay, op. cit., p. 9.

76 Op. cit., p. 19.

77 Op. cit., p. 61.

78 Op. cit., p. 30.
} 
sortent en quelque sorte d'une même pensée... tout découle d'un principe unique ${ }^{79}$. Lui-même parle de «pensée mère» dans son travail:

Ceux qui voudront y regarder de près retrouveront, je pense, dans l'ouvrage entier, une pensée mère qui enchaîne, pour ainsi dire, toutes ses parties ${ }^{80}$.

Il ajoute:

Il ne faut pas non plus oublier que l'auteur qui veut se faire comprendre est obligé de pousser chacune de ses idées dans toutes leurs conséquences théoriques, et souvent jusqu'aux limites du faux et de l'impraticable.

\section{Les traditions épistémiques nationales}

La notion d'identité collective est bien plus problématique encore que celle d'identité personnelle. C'est pourquoi je préfère utiliser la notion de tradition. Dans le domaine des idées politiques, par exemple, il est coutume de parler du pragmatisme des anglais-saxons et de l'amour des principes des français, avec, pour les premiers, le choix de la démocratie et l'acceptation des communautés comme fondement de la vie sociale, et, pour les seconds, le choix de la République (avec un $\mathrm{R}$ majuscule) et le refus d'un communautarisme qui mettrait en péril l'unité de la nation.

Dans le domaine philosophique, on opposera également l'empirisme anglosaxon aux philosophies continentales, attachées à faire ressortir les principes premiers. Dans le domaine des sciences mathématiques, on remarque la spécificité française du Bourbakisme, refondant les mathématiques sur des bases axiomatiques. On peut encore noter les apports respectifs des deux types de tradition au développement des statistiques. Ce sont les britanniques qui ont été à leur origine et leurs premiers développements, de façon empirique, cependant qu'on doit principalement aux français la création, dans un premier temps, du calcul des probabilités, puis, dans un deuxième, leur fondation rigoureuse, axiomatique (avec la participation des russes, dont on peut remarquer par ailleurs, en politique, qu'avec le soviétisme ils se sont fourvoyés à tenter d'appliquer certains principes politiques bien abstraits).

Ces schémas possèdent, naturellement, (et heureusement), des contreexemples: ainsi, la bataille mathématique célèbre, déjà évoquée, qui a opposé $H$. Poincaré et B. Russell, au sujet de la logique mathématique. C'est le français Poincaré qui s'élève contre le développement de cette discipline et se porte en défenseur de l'intuition, qu'il juge fondamentale en mathématiques également.

\footnotetext{
Les règles de la parfaite logique sont-elles toute la mathématique? Autant dire que tout l'art du joueur d'échec se réduit aux règles de la marche des pièces. Parmi toutes les constructions que l'on peut combiner avec les matériaux fournis par la logique, il faut faire un choix; le vrai géomètre fait ce choix judicieusement parce qu'il est guidé par un sûr instinct, ou par quelque vague conscience de je ne sais quelle géométrie plus profonde, et plus cachée, qui seule fait le prix de l'édifice construit ${ }^{81}$.
}

Lettre au comte Molé, 1835, op. cit., p. 11.

Op. cit., p. 71.

81 H. Poincaré, Les Mathématiques et la Logique, in Revue de métaphysique et de morale, 1905, p. 817; cité par A.-F. Schmid, op. cit., p. 149. 
Pour ce qui est de l'enseignement de la mécanique rationnelle, Poincaré se retrouve du côté de la tradition empirique anglaise.

Les Anglais enseignent la mécanique comme une science expérimentale; sur le continent, on l'expose toujours plus ou moins comme une science déductive et a priori. Ce sont les Anglais qui ont raison, cela va sans dire ${ }^{82}$.

J. Galtung est un sociologue et méthodologue norvégien original, à l'origine, un statisticien, qui suit sa propre voie dans une cité scientifique un peu trop étriquée pour sa curiosité insatiable. Il s'est amusé à proposer une classification de ce qu'il nomme «styles intellectuels», essai basé sur les impressions qu'il a glanées en fréquentant divers lieux internationaux du travail sociologique ${ }^{83}$. Ce sont des modèles, des «idéaux-types », qui montreraient une correspondance avec certains traits culturels, voire politiques, des pays en question.

Se refusant à établir une connexion trop forte entre le style et la nation, il utilise pour les désigner les termes de saxon, teuton, gallique - pour ne pas dire gaulois - et nippon.

Le saxon (essentiellement dans sa composante américaine) se veut avant tout opérationnel, il se basera sur des données, des documents, et se méfiera des envolées théoriques (au mieux, ira-t-il jusqu'à la «théorie de rang moyen » prônée par Merton). Corrélant ce style avec certains traits structurels des pays, Galtung remarque l'individualisme - l'encouragement à penser par soi-même - l'égalitarisme, la convivialité, qui permettent une discussion souvent «positive», encourageante, sur ces bases de consensus.

Le style teuton se veut déductif: il s'agit de tout déduire de quelques principes. Galtung note l'existence de ces «disciplines théoriques» que constituent le freudisme et le marxisme. Il remarque aussi les dangers d'une pensée pyramidale, qui porte en elle la croyance en des résultats supposés démontrés, à partir d'une base qui peut s'effondrer. Ici, le fond culturel est structurellement hiérarchique.

La France donne lieu à ce style apparemment inimitable d'un brillant théorique très proche de la littérature dont elle peut ainsi utiliser les armes de la séduction. Mais ce style littéraire peut aussi permettre d'aborder la complexité du réel dans des styles dialectiques, où les contraires sont représentés. On sait à quel point ce style de pensée a pu séduire en effet aux Etats-Unis les post-modernes qui cherchaient à se dégager du positivisme ${ }^{84}$. J'ajouterai que ce style, s'il est sans doute un des résultats de l'élitisme de l'enseignement français, à travers cette spécificité que sont les grandes écoles, n'est bien sûr pas le seul à exister ${ }^{85}$.

Dans le style nippon, la socialité est première, et vient avant le dialogue scientifique. Cette socialité est très hiérarchisée et collective, il s'agit avant tout de s'inscrire dans le sillage d'une autorité reconnue.

82 H. Poincaré, La Science et l'Hypothèse, Paris, Flammarion, 1932, p. 110.

83 J. Galtung, Structure, culture and intellectual style: An essay comparing saxonic, teutonic, gallic and nipponic approaches, in «Social Science Information», 20 (6), 1981, pp. 817-856. Je remercie J. Marcus-Steiff pour m'avoir fait connaître cet article.

84 J. Feldman, L'affaire Sokal, op. cit.

85 A. Barberousse, Le défi de la formation, in Angèle Kremer-Marietti, dir. Ethique et Epistémologie autour du livre «Impostures Intellectuelles» de Sokal et Bricmont, Paris, L'Harmattan, 2001, pp. 25-38. 


\section{Le problème du genre}

Si Galtung avait écrit son essai aujourd'hui, il aurait peut-être pris position sur le problème de l'identité épistémique sexuelle, soulevé ces dernières années par les féministes. Remarquons, pour continuer dans le cadre des observations de Galtung, que ce problème a surtout été posé dans les pays anglo-saxons - où, en effet, ont joué l'égalitarisme et le libéralisme - tandis qu'en France, l'élitisme et une réticence certaine à l'égard du féminisme ont relativement fait obstacle à ces nouveautés, jugées par ailleurs, souvent mal fondées.

Au départ de cette nouvelle problématique, un constat: la difficulté - prouvée par les statistiques - des femmes à arriver jusqu'aux postes les mieux reconnus de la cité scientifique. Il s'agit d'une ségrégation à la fois verticale (le prestige et le pouvoir), et horizontale (celle des disciplines, les femmes se retrouvant plus volontiers dans les sciences de l'homme que dans les sciences exactes) ${ }^{86}$.

Il fut un temps où l'explication dominante était l'incapacité foncière des femmes pour les études abstraites et la logique en particulier. Pour le «féminisme égalitaire» d'alors, il s'agissait donc de prouver que les femmes pouvaient faire aussi bien que les hommes. Puis, avec le changement de rapports de force produit par les mouvements de femmes des années soixante-dix et la critique de la science simultanée, un «féminisme différentialiste » est advenu, qui s'est posé la question de savoir si les femmes ne pourraient pas faire une science différente ${ }^{87}$.

Lorsque Galtung étudie les styles nationaux, il est en position d'extériorité sociologique classique, et ses travaux ont une influence minimale sur ces «faits sociaux ». Les études féministes, ou de genre, selon l'appellation, interviennent dans le cadre d'une sociologie idéologique et engagée. La mise à jour de la situation des femmes est, outre son caractère cognitif, et au vu des difficultés à se faire admettre pleinement par la cité scientifique, un acte quasi-militant pour la plupart des chercheuses et chercheurs de ce courant. Les explications proposées pour les statistiques observées se réfèrent à des philosophies dites «essentialiste» (qui défend l'existence d' «essences» masculine et féminine différentes) ou «universaliste» (qui prône la même réalité profonde pour les deux sexes) ${ }^{88}$. La position choisie intervient dans la définition même des identités sexuelles, en pleine évolution actuellement, elle appartient au fait social lui-même.

Pour ma part, j'estime importante la liberté pour chacun de se définir, laissant, en somme, à l'histoire en train de se faire les choix culturels des femmes et des hommes. C'est en me centrant sur la personne profonde que j'ai introduit le terme d'identité épistémique. Ce qui m'intéresse, c'est que chacun découvre la sienne, quelle qu'elle soit. C'est la raison pour laquelle j'ai des réticences envers les travaux qui concluent trop rapidement, en s'appuyant, soit sur des résultats biologiques controversés, soit sur des théories psychanalytiques bien vagues.

86 J. Feldman et A. Morelle, «Les femmes savantes» in France under the glass ceiling, in S. Lie, L. Malik, D. Harris, eds, The gender gap in Higher Education, World Yearbook of Education, London, Kogan Page, 1994, pp. 56-69.

87 S. Harding, The science question in feminism, Open University Press, 1986. J'ai écrit une critique de ce livre dans J. Feldman, Femmes, féminisme, science, in «L'Année Sociologique », 42, 1992, pp. 425-440.

88 Dictionnaire du féminisme, Paris, PUF, 2002. 


\section{Quelques axes}

Pour finir, je voudrais signaler quelques axes de l'identité épistémique concernant les sciences sociales, sans donner d'exemples, que chacun pourra facilement trouver. Dans chaque cas, ce qui fait «identité», c'est le choix privilégié ou le refus quasi-viscéral de l'approche, et c'est la raison pour laquelle ces caractéristiques apparaissent en couple et en opposition.

Commençons par l'axe de Pascal: finesse/géométrie, ou encore: complexitél simplicité. On «sent » plutôt la société, et on utilise un style impressionniste, littéraire, pour en rendre compte. Ou bien on aime plutôt en détacher quelques éléments jugés essentiels, ainsi que savent le faire les bons caricaturistes dans le dessin politique. On pourrait aussi utiliser le terme mathématique de première approximation, une première approximation qui parfois rend compte de la presque totalité de l'ensemble. Certains répugneront à cette approche, qu'ils jugeront simpliste, souffrant de voir abandonner le caractère complexe de la situation, alors même que ceux qui l'emploient ne sont pas dupes de la simplicité de leur modèle, qui est «résumé », mise en valeur de quelques traits. Le type-idéal de Weber appartient à cette approche.

Un deuxième axe est: concret/abstrait. Et certains sont plus à l'aise dans l'un ou l'autre registre. L'abstrait, ce serait par exemple d'envisager, en histoire, une vision sur la longue durée, ou de chercher des théories sociologiques vastes recouvrant de larges domaines du réel. Le concret, ce serait la clinique, l'empirie, le terrain, l'expérience. Il a été aussi évoqué, dans la section précédente, l'axe distanciation / engagement.

Un autre axe serait celui de la profondeurlétendue: choisir un domaine limité et le fouiller, approfondir une notion, un concept, ou alors tenter plutôt de couvrir largement, mais peut-être en surface seulement, un vaste domaine. Je cite ici Burton $(1577)^{89}$ :

Esprit inconsistant et volage, j'ai désiré toucher à tout, car je savais que je ne pouvais avoir plus qu'un talent superficiel dans chaque domaine. Savoir quelque chose dans tout, mais peu dans un domaine particulier, ce qui est le conseil de Platon - lequel soutenait qu'il ne faut pas être esclave d'une seule science, mais papillonner et «avoir une rame dans toutes les barques ».

\section{DIRE «JE» EN SCIENCE}

Quand le «je»se cache, notamment en sociologie et dans les sciences sociales, c'est une ruse honteuse!

Edgar Morin ${ }^{90}$

L’objectivité de la science a fait disparaître celui qui la fait, pourtant bien présent, et qui porte la responsabilité de ce qu'il publie en tant que résultats certains,

Cité par «Le Monde», 14 Avril 2000. R. Burton, L'anatomie de la mélancolie, José Corti, 2000.

90 E. Morin, Dialogue sur le sujet qui écrit, in «Cahiers Pierre-Baptiste», 13, décembre 1984, pp. 47-62. 
dits pour cela «scientifiques». D'autre part, un courant social modifie actuellement les règles de jeu qui délimitent le privé du public, poussant à la réapparition de ce «je» dont on se méfie tant en science. Le chercheur se trouve ainsi pris entre la tradition qui veut qu'il s'efface devant ses résultats et le désir ou besoin de prendre ses responsabilités et/ou d'affirmer sa part de subjectivité. «Dire je »- ou plutôt, dans le cas qui nous intéresse - «écrire je » n'est pas toujours simple. Il faut éviter divers écueils, celui de l'exhibitionnisme, par exemple, où l'on risque l'accusation, bien ou mal fondée, de narcissisme, ou d'arrogance... On a l'impression de s'exposer ${ }^{91}$. Dans l'examen qui suit, on commencera de nouveau par le cas des sciences exactes, on regardera du côté de la philosophie, avant d'aborder les sciences de l'homme.

\title{
Le cas des sciences exactes: $\mathrm{Tu}, \mathrm{je}$, nous
}

Dans ses débuts, la science n'est encore que «philosophie naturelle» et utilise donc son style. Dans l'Antiquité et au Moyen-âge, ainsi que le note J. Schlanger ${ }^{92}$, ce serait plutôt le «tu» qui est employé: on s'adresse au lecteur, on lui dit ce qu'il doit savoir, ce qu'il doit faire. Car le philosophe se trouve «du côté de la sagesse et du savoir vrai ». Voici, par exemple, au $13^{\text {eme }}$ siècle, Pierre de Maricourt, considéré comme le premier expérimentateur moderne, s'adressant directement à son lecteur, qu'il engage à refaire lui-même les expériences sur les aimants, afin d'être bien convaincu des effets qui sont décrits:

\begin{abstract}
Si tu veux voir ensuite comment la pierre attire une autre pierre, tu les prépareras toutes deux comme on l'a dit. Mets l'une dans un vase flottant..., tiens l'autre à la main et approche son pôle Nord du pôle Sud de la pierre flottante: celle-ci suivra alors la pierre que tu tiens comme si elle voulait y adhérer... Si tu présentes le pôle Sud de la pierre que tu tiens au pôle Nord de celle qui flotte... cette dernière suit celle que tu tiens. Nous connaissons donc la loi (regula) que le pôle Nord d'une pierre attire le pôle Sud d'une autre et le pôle Sud son pôle Nord. Si au contraire tu approches le pôle Nord du pôle Nord, tu verras la pierre qui flotte fuir sur l'eau celle que tu tiens et, de même, si tu approches un pôle Sud d'un pôle Sud, et cela parce qu'un pôle Nord désire un pôle Sud et fuit par conséquent un pôle Nord ${ }^{93}$.
\end{abstract}

A l'aube de la science moderne, on voit Galilée tenter de convaincre au moyen de dialogues entre trois personnages, celui qui porte ses propres vues, un opposant un peu borné, défenseur des traditions aristotéliciennes, et une tierce personne ouverte aux nouvelles idées.

La science moderne, à sa suite, insiste sur l'expérimentation et le recours au témoignage de ses propres sens. Au $18^{\text {ème }}$ siècle, où le scientifique travaille de façon encore isolée, un Dufay, qui découvre qu'il y a deux types d'électricité, expose soigneusement le déroulement de son expérience et ses résultats, en parlant à la première personne:

\footnotetext{
91 J. Feldman, Introduction, in J. Feldman et R. C. Kohn, dir., L'éthique dans la pratique des sciences humaines: dilemmes, Paris, L'Harmattan, 2000.

92 J. Schlanger, Gestes de philosophes, Paris, Aubier, 1994.

93 Epistola de Magnete (1269), cité par E. Bauer, L'électromagnétisme hier et aujourd' hui, Paris, Albin Michel, 1949, pp. 18-19.
} 
Ce qui me déconcerta prodigieusement fut l'expérience suivante: ayant élevé en l'air une feuille d'or par le moyen du tube, j'en approchai un morceau de gommé copal frotté et rendu électrique; la feuille fut s'y attacher sur-le-champ... J'avoue que je m'attendais à un résultat tout contraire parce que, selon mon raisonnement, le copal, qui était électrique, devait repousser la feuille qui l'était aussi; je répétai l'expérience un grand nombre de fois...

Après plusieurs tentatives qui ne me satisfaisaient nullement, j'approchai de la feuille chassée par le tube une boule de cristal de roche frottée et rendue électrique: elle repoussa cette feuille de même que le tube. Un autre tube de verre la chassa de même. Enfin je ne pus pas douter que le verre et le cristal de roche ne fissent précisément le contraire de la gomme copal, de l'ambre et de la cire d'Espagne, en sorte que la feuille repoussée par les uns à cause de l'électricité qu'elle avait, était attirée par les autres; cela me fit penser qu'il y avait peut-être deux genres d'électricité différents et je fus confirmé dans ces idées par les expériences suivantes...

Voilà donc deux électricités bien démontrées et je ne puis me dispenser de leur donner des noms différents... Que ne devons-nous pas attendre d'un champ aussi vaste qui s'ouvre à la physique; et combien ne nous peut-il fournir d'expériences singulières qui nous découvriront peut-être de nouvelles propriétés de la matière ? $^{94}$

Puis, une fois l'institution bien rôdée, l'anonymat devient de règle, à travers un «nous» collectif qui signifie: «Nous avons vu cela, obtenu tel résultat, et chacun pourrait également le voir, l'obtenir, en se plaçant dans les mêmes conditions que nous ». C'est la fameuse condition «Toutes choses étant égales par ailleurs» qui signale bien que l'on a réussi à isoler le phénomène en question. Il serait intéressant qu'une étude historique détaillée suive à la trace la disparition de la personne qui fait la science. Dans le corps du texte tout au moins. Car, le besoin de carrière l'exige, les articles sont bel et bien signés. Aujourd'hui, certaines expériences celles qui portent sur la matière sub-nucléaire, par exemple - se font dans des laboratoires immenses, avec une décomposition des tâches quasi-industrielle, et les articles sont signés par plusieurs dizaines de physiciens, appartenant à plusieurs laboratoires de différents pays.

Cette réduction de la personnalité du scientifique peut être mal vécue. Il se pourrait qu'elle soit une des raisons de la désaffectation qu'on remarque actuellement de la part des étudiants pour la science. E. Klein, physicien, tient une rubrique régulière dans La Recherche. "Quand les scientifiques parleront à la première personne», rêve-t-il dans une de ses chroniques ${ }^{95}$. Il y remarque que «l'imaginaire joue un rôle capital dans l'invention de nouvelles idées», alors que «la rigueur, elle, corrige les mauvais tirs, mais ne crée pas». On aborde donc bien ici les séparations établies dans la société entre public et privé. Les physiciens entre eux, s'ils sont amis, peuvent se livrer l'un à l'autre intimement, mais l'image officielle qu'ils doivent donner dans leurs publications, est celle de l'objectivité et de la rigueur.

\section{L'usage du «je» par les philosophes}

Le livre de J. Schlanger Gestes de philosophes s'attache à examiner l'activité philosophique sous un angle neuf, qui me paraît appartenir au courant que j'ai

\footnotetext{
94 Cité par Edmond Bauer, op. cit., p. 47.

95 «La Recherche», n² 280, octobre 1995.
} 
mentionné. Bien qu'insistant sur le fait que des éléments importants de sa pensée prennent racine dans la personnalité profonde du philosophe, Schlanger n'entend pas ramener le travail philosophique à une subjectivité pure. En effet, la philosophie consiste avant tout en un échange des pensées, et pas seulement, comme l'œuvre d'art, en une production au monde qui trouve sa fin en soi. Le seul fait qu'il soumette son travail à la discussion rationnelle implique que le philosophe tend vers un type d'universalité. L'auteur définit avec précision la spécificité de son regard: il ne s'agit pas de sonder dans la personnalité du philosophe pour trouver les raisons de sa pensée, mais d'examiner ce qu'il nomme une «gestuelle», c'est-à-dire des façons de présenter cette pensée.

Par «gestuelle», j'entends ici tout ce qui, dans une production d'objets idéels théoriques, n'est pas à proprement parler théorique, sans pour autant se réduire au psychologique et au social ${ }^{96}$.

Dans cette «gestuelle», l'usage du «je» tient une place importante. Car avec la venue de la science moderne, qui a trouvé les moyens de la preuve, la philosophie devient moins sûre d'elle même et le philosophe doit admettre qu'il ne peut faire reposer la validité de sa vérité que sur lui-même.

Avec l'amplification du questionnement sceptique, avec l'usage du doute pour fonder la certitude, on ne peut partir que de soi: et parler de soi se présente ainsi comme un signe marquant de la modernité ${ }^{97}$.

Mais en parlant de soi, le philosophe vise à la généralité.

Il n'use de soi qu'autant que cela lui paraît important pour les besoins de son intention, pour les idées qu'il cherche à développer. Son «soi» lui sert de matériau, et parler de soi n'est pas pour lui l'occasion d'une complaisance ${ }^{98}$.

Schlanger distingue alors plusieurs rôles remplis par ce «je». Il s'agit essentiellement de trois modes, qu'il nomme paradigmatique, existentiel et transcendantal. Même si dans la pratique, ces modes interfèrent, l'illustration de chacun par le nom d'un philosophe permet déjà de s'en faire une idée: "Montaigne use plutôt du mode paradigmatique, Kant du mode transcendantal, Kierkegaard du mode existentiel.»

Le mode existentiel est évidemment celui de Kierkegaard, ce philosophe qui a publié sous divers pseudonymes, comme à la recherche constante de sa propre identité. C'est encore celui de Nietzsche, qui, délaissant le pur déroulement du raisonnement, livre ses colères et ses invectives. Ici, l'affect se mêle à la pensée philosophique, dans un romantisme qui rompt avec l'approche classique froide, distanciée, contrôlée. Dans ce désir de saisir une singularité, Schlanger voit l'œuvre d'un artiste: l'usage de ce mode rejoint l'esthétisme.

Le mode philosophique le plus classique est le transcendantal, et ce sont Kant, Husserl, Merleau-Ponty qui sont convoqués ici, mais aussi le Descartes du cogito. Le philosophe transcende la réalité pour arriver à des vérités générales, et s'ef-

\footnotetext{
J. Schlanger, Gestes de philosophes, op. cit., p. 7.

Op. cit., p. 16.

8 Op. cit., p. 23.
} 
force «d'agir à la manière du chercheur scientifique». Le «je» est alors « un 'Je' essentiel sur lequel vient se greffer notre particularité $»^{99}$.

Reste le mode paradigmatique, représenté par Montaigne, qui décide de se décrire, afin de décrire l'humanité entière. C'est la fameuse formule: «chaque homme porte la forme entière de l'humaine condition ». Dans le concret de la description, dans la minutie d'un cas particulier, Montaigne vise à toucher la vérité même de l'être humain, dans une accumulation de notes, sans souci de généraliser. On sait que son entreprise, si elle séduit les uns, en agace d'autres : «le sot projet qu'il eût de se peindre», estimait Pascal.

Voilà, pour illustrer ces modes, certains passages de Descartes : lorsqu'il commence l'énoncé de ses règles du discours de la méthode par un récit de ce que l'on nommerait aujourd'hui, une autobiographie intellectuelle, il est dans le mode «existentiel». Mais peu à peu, à partir de son propre exemple, il va passer au «je paradigmatique »: ce qu'il propose est valable pour d'autres aussi, même s'il feint de poser qu'il ne fait que montrer son «modèle», mais ne cherche d'aucune façon à être imité. «Le 'Je' de l'autobiographie intellectuelle devient un 'Je' de l'injonction cognitive». Puis, en continuant son trajet de pensée, Descartes aborde le «je transcendantal», celui qui a fait rupture avec les réalités contingentes pour se mouvoir dans le domaine de la pensée pure.

Le mode paradigmatique possède un aspect pédagogique: il s'agit d'enseigner, de mieux faire comprendre, non pas pour que nous imitions le philosophe, mais pour que nous-mêmes nous soyons en mesure de nous interroger sur nousmêmes et d'apprendre à mieux nous connaître, sur le plan de la vie pour Montaigne, sur le plan de la méthode de pensée pour Descartes.

«Apprendre à vivre en contemplant d'autres vies, n'est-ce pas là le fondement du prêche éthique et religieux, de l'édification fondée sur l'imitation », remarque encore Schlanger. Aussi, si les deux premiers modes touchaient au beau, ou au vrai, celui-ci toucherait plutôt au bien.

\section{Le cas des sciences sociales}

En m'inspirant du travail de Schlanger, je vais proposer de nommer quelquesuns des modes d'utilisation du «je»dans les sciences sociales. Et comme lui, il faut rappeler qu'il s'agit là d'idéaux-types, qui peuvent se mélanger les uns aux autres, et/ou être utilisés de façon successive.

\section{Le «je» comme instrument de connaissance}

On pourrait dire qu'il est «instrumental», mais le terme a des connotations indésirables ici. Le chercheur s'implique pour connaître, il s'utilise lui-même en tant qu'instrument de connaissance.

Tel est le cas, spectaculaire, de J. Favret-Saada dans son étude sur la sorcellerie, qui a déjà été mentionnée. Elle est prête à s'engager dans une aventure personnelle pour connaître, et c'est cet engagement même, le fait d'avoir été «affectée», selon le terme qu'elle a choisi, qui lui permet de faire avancer la connaissance.

99 Op.cit., p. 19. 
Un autre exemple est celui du couple de sociologues M. Pinçon et M. PinçonCharlot, couple également à la ville. Leur livre Voyage en grande bourgeoisie, Journal d'enquête ${ }^{100}$ relate leur expérience de recherche, en traitant «la chaîne méthodologique» dans son ensemble, en «dévoilant» les «arcanes du métier de chercheur», les «secrets de fabrication» de leurs études. Ils parlent d'un «travail d'auto-analyse épistémologique» en affirmant: «pas de science du social sans socioanalyse du sociologue». Ils notent, par exemple, qu'être un couple marié représentait une respectabilité qui les a aidés à se faire inviter et accepter dans un milieu très fermé.

\begin{abstract}
Ce «nous» (double personne du singulier) nous engage comme producteurs de la recherche. Il nous a semblé que nous devions ainsi nous exposer dans cette tentative pour restituer les dimensions affectives, incontournables, du rapport du chercheur en sciences sociales, donc humaines, aux objets - humains - de son investigation. Or ce n'est pas là un mode d'écriture académique car dans «son rapport à l'institution de recherche universitaire... l'écriture en sciences sociales se construit un statut en rejetant, autant que possible, les implications les plus intimes $»^{101102}$.
\end{abstract}

Vient alors l'inévitable évocation d'un narcissisme dont on veut se démarquer et dont on craint d'être accusé: «au-delà du possible narcissisme, inconscient, d'une telle attitude $» . .$.

\title{
Le «je» existentiel
}

R. Lourau, qui vient d'être cité, est un de ceux qui tiennent à faire apparaître le rôle de la subjectivité dans les sciences sociales. Il insiste auprès de ses étudiants sur la tenue et la publication des «journaux de recherche», ainsi que sur la notion d'implication. Dans ses livres, il entremêle souvent au texte proprement dit des extraits de son journal. Le contenu de ces extraits de vie privée est le plus souvent disjoint de la pensée développée dans le texte. Tout se passe comme si Lourau, témoignant de son refus d'une apparente coupure entre le travail intellectuel et la vie, tenait à faire apparaître l'auteur dans son concret quotidien, et non seulement à travers le déroulement de ses pensées. Ce serait comme un besoin de se faire connaître, dans le même sens, mais en allant plus loin, que la photo que nombre d'éditeurs tiennent à donner d'un auteur. Lourau nous fait part de ses doutes, la recherche sur l'implication s'avère difficile, et il évoque son «exhibitionnisme de timide ${ }^{103}$. Je parlerai ici d'un «je» existentiel, qui se donne à voir, à côté du je «transcendantal» qui prétend à un discours vrai.

100 M. Pinçon et M. Pinçon-Charlot, Voyage en grande bourgeoisie, Journal d' enquête, Paris, PUF, 1997.

101 Est cité ici René Lourau, Le Journal de recherche. Matériaux pour une théorie de l'implication, Paris, Editions Méridiens/Klincksieck, 1988, p. 15.

102 Voyage en grande bourgeoisie..., op. cit., p. 118.

103 R. Lourau, Le lapsus des intellectuels, Privat, 1981. Dans ce livre est reproduit, par exemple, le contrat avec son éditeur. J'ai analysé ce livre dans G. Monceau, A. Lamihi, dir., Institution et implication. L' œuvre de René Lourau, Syllepse, à paraître. 


\section{Le «je» paradigmatique}

G. Monceau est un élève de Lourau. Il a soutenu sa thèse sur la notion de «résistance ${ }^{104}$. Il nous fait participer à la construction de cette thèse et de cette notion, à travers les dédales de l'apprenti chercheur. Je nommerai le récit de ce parcours un «je » paradigmatique, puisqu'il engage d'autres à faire de même, dans un optique où, comme le demandait par exemple Marrou, les éléments de la construction d'un savoir sont soumis honnêtement au lecteur, en refusant les sauts qu'accepterait sans doute un partisan de Popper et de ses trois mondes.

De par la diversité des sciences sociales, de leurs objets, de leurs approches, un récit d'un trajet de connaissance possède, à mon avis, une validité en soi-même, en montrant, sur un cas particulier, comment se déroule l'interaction, de nature cognitive, entre le chercheur et ses objets d'étude, qu'il construit peu à peu, en les faisant sortir du magma social primaire.

C'est dans ce mode que je situerai aussi le texte de P. A. Adler et P. Adler ${ }^{105}$, qui nous parlent de l'autocensure dans les sujets sensibles. A travers leurs expériences de recherche, ils nous livrent quelques résultats théoriques et méthodologiques qui peuvent servir à d'autres.

\section{Le témoignage sociologique}

Une autre manière de se mettre en jeu est ce que je propose de nommer le témoignage sociologique. On utilise le fait que le sociologue appartient à la société, et que le fait qu'il y vive lui donne un savoir qui peut être pertinent, même si ce savoir - comme tout savoir, par ailleurs - peut connaître des limites, ici cette fameuse proximité, objet des soupçons de ceux qui choisissent la posture de distanciation. Par ailleurs cette distanciation existe bien évidemment, elle consiste à repérer dans une aventure de vie aux informations surabondantes la composante sociale qui l'a animée. Ainsi, J.-L. Le Grand a soutenu sa thèse en décrivant et analysant une expérience de vie communautaire qu'il a faite dans les années postmai $68^{106}$. R. Hess défend la pratique du journal comme une façon de décrire des parties de la réalité sociale ${ }^{107}$. Il m'est arrivé d'utiliser moi-même quelques morceaux de ma vie pour illustrer des changements sociaux où j'étais à la fois agent et $\operatorname{agie}^{108}$.

On peut encore citer, parmi les ouvrages fondés sur l'expérience personnelle, venue de l'autre côté de la Manche et qui a été très bien accueillie, l'autobiogra-

104 G. Monceau, Le Concept de résistance en éducation. Conceptualisation descriptive et opératoire, Thèse de Doctorat, Université de Paris VIII-Vincennes, 1997.

105 P. A. Adler et P. Adler, L'autocensure dans les sujets sensibles, in J. Feldman et R. C. Kohn, dir., L'éthique..., op.cit., pp. 163-180.

106 J.-L. Le Grand, Etude d'une expérience communautaire à orientation thérapeutique, Histoire de vie de groupe. Perspectives sociologiques. Thèse pour le doctorat d'Etat ès Lettres et sciences humaines, Université Paris VIII, 1987.

107 R. Hess, La pratique du journal. L'enquête au quotidien, Paris, Anthropos, 1998. Et aussi: Le lycée au jour le jour, Ethnographie d' un établissement d'éducation, Paris, Méridiens Klincksieck, 1989.

108 Par exemple, J. Feldman, Valeurs, Science, Institution : une traversée en trois temps, in J. Feldman et R. Kohn, dir. L'éthique :, op.cit., pp. 131-159. 
phie de R.Hoggart, un professeur de littérature. C. Grignon, dans son introduction, place cette œuvre parmi les ouvrages sociologiques majeurs ${ }^{109}$.

\section{La formation de soi; le praticien-chercheur}

Agents de la société et, en même temps, agis par elle, nous le sommes tous. La prise de conscience de ce phénomène me semble appartenir, dans l'espace moderne, à ce «souci de soi » issu de la philosophie ancienne et dont nous parle Foucault ${ }^{110}$. La flèche de la connaissance, qui part de soi pour aller vers une appréhension de la société, revient ici, ensuite, sur soi-même. La prise en considération des aspects sociologiques de son existence correspond à une prise de distance par rapport à cette vie, une vie examinée, relativisée, mieux maîtrisée, mieux comprise. Certaines pratiques de formation à l'Université visent cette prise de conscience, médiatisée par la recherche et la connaissance de type universitaire. H. Desroche, un des pionniers de cette démarche, soucieux d'établir des ponts entre l'Université et le monde civique, avait créé un «collège coopératif », sur le modèle du compagnonnage. Là, il engageait ses étudiants, venus du monde du travail, à parler d'eux. Le passage par la réflexivité est considéré comme une «maïeutique» qui permet de mieux formuler ensuite son objet d'études ${ }^{111}$. Ce recours aux histoires de vie est une démarche ancienne, comme le montre $\mathrm{C}$. Delory-Momberger ${ }^{112}$. La notion de «praticien-chercheur», proposée par R. C. Kohn, et défendue à son tour par A. Perrault-Solivères ${ }^{113}$, concerne des personnes actives qui viennent à l'Université pour prendre du recul, et acquérir les moyens d'une réflexion sur leur démarche professionnelle, et aussi sur eux-mêmes. Il peut être alors difficile d'établir la frontière entre la recherche existentielle, personnelle, et la recherche savante. On rencontre bien évidemment là une des spécificités majeures des sciences de l'homme, qui appelle à des expériences et des réflexions qui ne font que commencer.

\section{Des expériences de groupe}

Tenter de trouver ce qui, en soi, conduit à percevoir certains aspects de la réalité sociale plutôt que d'autres, ce qui porte à choisir aussi les modes d'approche, tenter de dégager quelles sont ses propres voies de connaissance, ces questions me paraissent importantes à dégager, aussi bien pour soi-même que pour la discipline que l'on veut servir. Se poser ces questions en groupe permet à chaque participant une prise de conscience, qui réagit à celle des autres, toute identité se révélant d'autant mieux qu'elle a à se situer par rapport à celle des autres. Ce mode de com-

109 R. Hoggart, 33 Newport Street; Autobiographie d'un intellectuel issu des classes populaires anglaises. Paris, Gallimard, Le Seuil, 1991.

110 M. Foucault, L'Herméneutique du Sujet, Paris, Gallimard/Le Seuil, 2000. Je remercie E. Touton de m'avoir introduite à cet ouvrage.

111 H. Desroche, Entreprendre d'apprendre. D’une autobiographie raisonnée aux projets de recherche-action. Paris, Ed. ouvrières, 1990, p. 97.

112 C. Delory-Momberger, Histoires de vie, De l' invention de soi au projet de formation, Anthropos, 2000.

113 A. Perrault-Solivères, Infirmières, le savoir de la nuit, Prix Le Monde de la recherche universitaire, Paris, PUF, 2001. 
munication, où la recherche d'un échange sincère passe avant tout jugement de valeur, a été rendu assez familier par les pratiques psychothérapeutiques de groupe qui se sont répandues dans la société. Il y faut une grande confiance entre les participants, laquelle n'existe par ailleurs pas facilement dans des institutions soumises aux lois de la compétition et du jugement.

J'évoquerai ici l'expérience du groupe clinique dont R. C. Kohn est à l'origine, et qui a été décrite par S. Schaepelynck-Boniface dans sa thèse ${ }^{114}$. Elle en souligne la nouveauté des pratiques d'échange, en rupture avec les façons habituelles du travail académique, la recherche et la prise de conscience de nouveaux modes d'appréhension de ces «objets sociaux» en perpétuel déplacement, la nécessité de styles appropriés pour en rendre compte.

\section{Le «je» de la maîtrise}

Etre capable d'utiliser le «je» de façon juste, sans crainte de déraper dans le narcissisme ou l'exhibitionnisme, se produit lorsque l'auteur possède une double maîtrise, celle de son langage professionnel et celle de son moi personnel. Ces maîtrises se gagnent au cours du temps, aussi, c'est le «Maître» chevronné, reconnu, qui pourra s'y livrer sans risque.

Je donnerai ici l'exemple de l'économiste Malinvaud, tel qu'il a été relevé par F. Lordon ${ }^{115}$. La critique qu'il fait de la prétention à la scientificité de l'économie mathématique porte d'autant mieux qu'il est de ceux qui ont contribué à développer cette discipline en France ${ }^{116}$. C'est en tant que «sage», au risque de paraître «ennuyeux», voire, «déplaisant», dit-il, qu'il aborde cette critique. Le «je» est ici comme la présentation de sa très grande légitimité et de son engagement responsable dans son propos. Le fait d'utiliser le «je» donne plus de poids à sa position que ne le ferait la simple discussion épistémologique qu'il offre par ailleurs. Car c'est lui-même, avec son expérience et ses compétences, qu'il engage dans le combat.

P. Nora avait demandé, il y a quelque temps, à des historiens reconnus de parler à la première personne, de proposer leur «ego-histoire ${ }^{117}$. Chacun devait se présenter dans son travail professionnel. Le titre «ego» apparaissait comme une sorte de provocation à l'objectivité supposée de l'historien et comme un défi à l'accusation de narcissisme. Dans sa préface, Nora propose «un genre nouveau, pour un nouvel âge de la conscience historique». Face à cette ambition, le résultat s'avère quelque peu décevant, comme le reconnaît Nora lui-même: ces «récits de vie» d'historiens reconnus servent à les faire connaître davantage, certes, mais n'introduisent pas vraiment de genre nouveau de connaissance.

\footnotetext{
114 S. Schaepelynck-Boniface, Démarche Clinique. In L'Orientation dans l'entre-deux des motifs. Espaces de rencontres avec des lycéens de seconde en situation-limite. Thèse de Doctorat, Université Paris VIII-Saint-Denis, 2000.

115 F. Lordon, Le désir de faire science in «Actes de la Recherche en Sciences Sociales», 119, 1997, pp. 27-35.

116 E. Malinvaud, Pourquoi les économistes ne font pas de découvertes in «Revue d'économie politique», 106, 1996, pp. 929-942.

117 P. Nora, Essais d' ego-histoire, Paris, Gallimard, 1987.
} 
Notons qu'il est habituel de vouloir mieux connaître la vie des personnes qui ont atteint une grande notoriété, débordant de leur milieu professionnel. C'est ainsi qu'Einstein, qui disait n'avoir pas beaucoup l'habitude de parler de lui, avait fini par accepter d'écrire son autobiographie, qu'il avait d'ailleurs vite dévié vers son trajet intellectuel.

Dans ses «Lettres à une étudiante», Touraine, ayant bien acquis la reconnaissance de son statut, reconnaît que ce type d'écriture, qui est ici conversation, le fait avancer dans sa pensée. Il nous livre, au passage, cette confession:

J'envie ceux qui se sont trouvés très tôt maîtres de leur pensée ou de leur action. Je me délivre lentement et presque par force de tout ce qui m'empêche de me trouver ${ }^{118}$.

\section{Les risques $d u$ «je»}

Bien plus difficile et risqué représente, pour des personnes moins «autorisées », le fait de quitter le style impersonnel habituel au champ académique, produit des traditions scientifique et philosophique. Plusieurs de ceux qui s'y essaient font état de leur embarras.

Après l'ego-histoire, voilà, dans une discipline traditionnellement proche, une ego-géographie tentée par J. Lévy, qui sous-titre: «matériaux pour une biographie cognitive». Cet auteur examine le déroulement de sa pensée et de son travail de géographe, face aux évolutions de sa discipline. Il est conscient des difficultés de son entreprise, où il s'est engagé par «goût du risque». Les deux premières phrases de la quatrième de couverture expliquent: «Les chercheurs hésitent à parler d'eux-mêmes. Ils craignent, non sans raison, de sortir de leur compétence et de sombrer dans un narcissisme ennuyeux ». Il expose, au début de son livre ses scrupules:

Parler de soi est tentant et dangereux. Ce peut être utile pour soi-même, éventuellement pour les autres, à certaines conditions... Je ne suis pas sûr d'avoir su éviter les écueils que ce type d'entreprise fait surgir ${ }^{119}$.

J. Lévy a déjà un trajet professionnel reconnu quand il se risque à l'aventure. Tel n'est pas le cas de F. Weber qui livre dans sa thèse « des éléments subjectifs par souci d'objectivité ${ }^{120}$. Elle a été encouragée à le faire par son Directeur de Thèse, G. Althabe, qui trouve même qu'elle n'en dit pas assez, mais elle a suscité quelques réactions très négatives dans son milieu. Elle avoue qu'elle a été inconsciente de «l'ampleur des risques», se retrouvant seule dans cette aventure non balisée. Pourtant, il apparaît qu'elle n'est pas si seule, pas vraiment démunie, puisqu'elle dit tenir d'abord à l'opinion d'un petit nombre de personnes qu'elle apprécie.

Je terminerai, en remontant le temps, avec le sociologue américain A. W. Gouldner. Professeur reconnu, titulaire d'une chaire portant le nom de Max

118 A. Touraine, Lettres à une étudiante, Paris, Seuil, 1974, p. 82.

119 J. Lévy, Ego-géographies; Matériaux pour une biographie cognitive, Paris, L'Harmattan, 1995, p. 10.

120 G. Noiriel, entretien avec Florence Weber, Journal de terrain, journal de recherche et auto-analyse in «Genèses », 2, 1990, pp. 138-147. 
Weber, ayant des opinions de gauche, il a été déconcerté par la révolte des étudiants des années soixante, qui remettent en cause la valeur de la sociologie. Gouldner réfléchit alors sur la «crise de la sociologie occidentale». Sa solution à cette crise: une sociologie réflexive, où le sociologue s'engage en tant qu'être humain, et apprend à se connaître lui-même et à expliciter ses propres valeurs. «La qualité du travail du sociologue dépend de sa qualité en tant qu'être humain », écrit-il ${ }^{121}$.

L'engagement de l'auteur reste pourtant, la plupart du temps, sur les sommets théoriques, et c'est avec une sorte de défi à lui-même qu'il semble lancer enfin sa personne même dans la bagarre, page 482 d'un livre qui en contient 512 . Il parle tout d'abord de «Gouldner», comme si le «je» était impossible à prononcer. C'est après la septième utilisation de «Gouldner» qu'il se sent en mesure de glisser enfin au pronom personnel «my» («Gouldner's efforts, my efforts», écrit-il, souligné par moi). Moment dramatique, qui illustre la difficulté, évoquée plus haut, pour le scientifique, de passer au «je».

\section{CONCLUSION}

On sait que Comte, ce penseur puissant et excessif, qui se trouve être le créateur du mot «sociologie», a eu deux périodes dans son activité intense. La première est «objective», il s'agit de trouver les «lois» scientifiques qui conduisent la civilisation, et d'aboutir, après les sciences constituées - mathématique, physique, biologie - et en utilisant la méthode «positive», à la sociologie, « reine des sciences », qui va permettre de gérer la société de façon «positive».

Puis se produit une rencontre amoureuse d'une importance extrême. A partir de là, Comte va mettre l'accent sur la «subjectivité », c'est-à-dire les sentiments, les affects, mais aussi la spiritualité. Ceci ne représente d'ailleurs pas pour lui, qui tient au holisme de sa pensée, une rupture avec ses travaux précédents, mais plutôt une continuation, un dépassement, comme un apogée, qui le conduit à définir la nouvelle religion, positive, de l'humanité. C'est, dit-il, dans la mesure où la sociologie a été fondée par lui, qu'on se trouve en mesure d'en arriver à une appréhension «positive» de la subjectivité ${ }^{122}$.

Comte représente ainsi, à sa manière exagérée, à la fois l'enthousiasme pour la science du $19^{\text {ème }}$ siècle, mais aussi un romantisme aux passions sublimées dans la création d'une religion, le tout présenté comme faisant partie du même système, le positivisme. Que ce serait-il passé si la psychanalyse avait existé de son temps et qu'il ait décidé d'en tâter?

En effet, un demi-siècle plus tard, un médecin autrichien, S. Freud, commençait une exploration des mondes intérieurs des personnes, en se revendiquant constamment de la science, et en prenant ses distances avec la religion et le mys-

121 A. W. Gouldner, The Coming Crisis of Western Sociology, New York, London, Basic Books, 1970, p. 494. Traduction du dernier chapitre, dont il est question ici, dans la «Revue du Mauss », 1989, $\mathrm{n}^{\circ} 4$.

122 R. Lourau, La question du sujet chez Auguste Comte, in «L'homme et la société», 1991, n 101, pp. 57-68. A. Kremer-Marietti, La méthode subjective, in M. Bourdeau et Fr. Chazel, dir., Auguste Comte et l'idée d' une science de l' homme, L'Harmattan, 2002. 
ticisme. C'est ainsi qu'un apprivoisement de type rationnel des mystères de la subjectivité profonde a pu être entamé - qui aurait peut-être pu aider Comte à mâ̂triser autrement ses pulsions qu'en les sublimant dans des constructions intellectuelles exaltées.

Au cours de ce texte, des auteurs très rationnels ont été cités au sujet du rapport personnel à la démarche scientifique. Nous avons entendu l'historien des sciences Koyré évoquer, pour ses débuts, une révolution spirituelle, le psychologue américain positiviste Rogers avouer un quasi-mysticisme, le mathématicien Poincaré défendre le rôle de l'intuition, Bourdieu lui-même en arriver à l'exercice spirituel, sans compter les blessures identitaires relevées dans l'affaire Sokal. Ceci souligne à quel point la recherche d'une connaissance exigeante - dans notre cas, celle qui se veut scientifique - est une aventure qui demande un investissement du chercheur dans des dimensions qui peuvent dépasser l'intellectualisme et la rationalité purs. Or la recherche de vérité, de connaissance profonde, appartiennent également aux démarches religieuses. Pas étonnant alors que le scientisme, qui veut refuser les autres démarches et ne considère comme valable que celle de la science, ait pu prendre la place, comme il a souvent été remarqué, de la religion, en en mimant les aspects les plus fermés.

La plus grande familiarité avec notre monde intérieur qui est proposée dans notre société incite à ce que soient mieux examinées, au plan personnel, les façons de connaître dans les domaines qui se veulent relever de la scientificité, surtout quand celle-ci est loin d'être évidente. C'est à la fois un enrichissement pour le chercheur et pour la discipline, si on évite les dérapages qui, comme pour toute démarche, peuvent exister, et doivent être signalés. Cet article avait pour but de faire ressortir quelques-unes des implications des chercheurs dans leurs sciences: plus faciles à décrire dans les sciences objectives, parce que la personnalité du chercheur y est surtout réduite à ce qui les concerne; plus difficile et risquée dans le cas des sciences de l'homme, où l'intervention de la personnalité est bien plus étendue. C'est pourtant là qu'il serait le plus nécessaire de reconnaître et d'accepter l'existence de cette intervention et d'en repérer quelques modalités. C'est ce qui a été tenté ici.

CNRS - GEMAS

Maison des sciences de l' homme, Paris 